\title{
Entanglement dualities in supersymmetry
}

\author{
Robert H. Jonsson $\odot,{ }^{1, *}$ Lucas Hackl $\odot, 2,3, \dagger$ and Krishanu Roychowdhury $\oplus^{4, \$}$ \\ ${ }^{1}$ Max-Planck-Institut für Quantenoptik, Hans-Kopfermann-Straße 1, 85748 Garching, Germany \\ ${ }^{2}$ School of Mathematics and Statistics \& School of Physics, The University of Melbourne, Parkville, Victoria 3010, Australia \\ ${ }^{3}$ QMATH, Department of Mathematical Sciences, University of Copenhagen, Universitetsparken 5, 2100 Copenhagen, Denmark \\ ${ }^{4}$ Department of Physics, Stockholm University, SE-106 91 Stockholm, Sweden
}

(Received 22 March 2021; revised 6 May 2021; accepted 7 May 2021; published 16 June 2021)

\begin{abstract}
We derive a general relation between the bosonic and fermionic entanglement in the ground states of supersymmetric quadratic Hamiltonians. For this, we construct canonical identifications between bosonic and fermionic subsystems. Our derivation relies on a unified framework to describe both bosonic and fermionic Gaussian states in terms of so-called linear complex structures $J$. The resulting dualities apply to the full entanglement spectrum between the bosonic and the fermionic systems, such that the von Neumann entropy and arbitrary Renyi entropies can be related. We illustrate our findings in one- and two-dimensional systems, including the paradigmatic Kitaev honeycomb model. While typically supersymmetry preserves features like area law scaling of the entanglement entropies on either side, we find a peculiar phenomenon, namely, an amplified scaling of the entanglement entropy ("super area law") in bosonic subsystems when the dual fermionic subsystems develop almost maximally entangled modes.
\end{abstract}

DOI: 10.1103/PhysRevResearch.3.023213

\section{INTRODUCTION}

As a long-established concept in quantum physics, supersymmetry (SUSY) finds applications in a wide range of fields from particle physics to condensed matter in both relativistic and nonrelativistic settings [1-5]. In a nutshell, SUSY posits a fundamental equivalence between the two classes of elementary particles with distinct statistics. Mathematically, it maps the fermionic degrees of freedom to the bosonic ones and vice versa. From this perspective, they are equivalent and dubbed superpartners of each other.

While normally SUSY is conceived as a symmetry in quantum field theories, it as well applies to much simpler models of quantum mechanics such as harmonic oscillators or the hydrogen atom [6-9]. The SUSY Hamiltonian $\hat{H}$ can be constructed from a generating operator $\hat{\mathcal{Q}}$ (also called the supercharge operator) which, for the harmonic oscillator problem, takes a remarkably simple form $\hat{\mathcal{Q}}=\sqrt{\omega} \hat{b}^{\dagger} \hat{c}$, where $\hat{b}(\hat{c})$ denotes the bosonic (fermionic) annihilation operator. The corresponding SUSY Hamiltonian

$$
\hat{H}=\left\{\hat{\mathcal{Q}}, \hat{\mathcal{Q}}^{\dagger}\right\}=\omega\left(\hat{b}^{\dagger} \hat{b}+\hat{c}^{\dagger} \hat{c}\right) \equiv \hat{H}_{\mathrm{b}}+\hat{H}_{\mathrm{f}},
$$

then decomposes into two simple quadratic Hamiltonians: one for a bosonic oscillator $\left(\hat{H}_{\mathrm{b}}\right)$ and the other for a fermionic one

\footnotetext{
*robert.jonsson@mpq.mpg.de

†lucas.hack1@unimelb.edu.au

*krishanu.1987@gmail.com
}

Published by the American Physical Society under the terms of the Creative Commons Attribution 4.0 International license. Further distribution of this work must maintain attribution to the author(s) and the published article's title, journal citation, and DOI. Open access publication funded by the Max Planck Society.
$\left(\hat{H}_{\mathrm{f}}\right)$. When it comes to dealing with real bosons or fermions, a Hermitian form of the generating operator $\hat{\mathcal{Q}}=\hat{\mathcal{Q}}^{\dagger}$ (and accordingly, $\hat{H}=\hat{\mathcal{Q}}^{2}$ ) is useful, as also is the case for this paper.

Such a simple setting is readily amenable to accommodate multiple bosonic and fermionic modes, or in other words, systems of free (noninteracting) bosons and fermions (in the continuum or on a lattice) if the generating operator $\hat{\mathcal{Q}}$ involves the bosonic and fermionic operators to linear order $[10,11]$, as shown in the previous harmonic oscillator example and also will be demonstrated later. The resulting partner Hamiltonians (referred to as $\hat{H}_{\mathrm{b}}$ and $\hat{H}_{\mathrm{f}}$ for bosons and fermions, respectively) are isospectral in their one-particle excitations except for zero modes. Inclusion of zero modes in SUSY has, in addition, a topological aspect (referred to as "Witten index" [12] and interpreted in several other contexts, e.g., see Ref. [13]) and has been studied to a great extent; however, that discussion is not relevant to this paper.

Ground states of a quadratic Hamiltonian (bosonic or fermionic) garner special attention, as they provide a fertile ground to trace several properties of the system, which they are part of, analytically. These states are also known as Gaussian states [14-18]. The study of the von Neumann bipartite entanglement entropy plays a central role in the quantum foundations of statistical mechanics [19-35], in quantum information theory [36-45], and condensed matter dedicated to classifying novel states of matter, particularly those with topological quantum order [46-54]. While measuring entanglement is numerically costly for a generic quantum state, it greatly simplifies for the Gaussian states [55,56].

The main result of this paper is a duality between the eigenvalues of reduced density operators in the bosonic and the fermionic system, i.e.,the so-called entanglement spectra. For Gaussian states, these spectra are fully encoded in the 
eigenvalues $\pm \mathrm{i} \lambda$ of the so-called restricted complex structure $J$, where $\lambda_{\mathrm{b}} \in[1, \infty)$ for bosons and $\lambda_{\mathrm{f}} \in[0,1]$ for fermions. In supersymmetric systems, the charge operator $\hat{\mathcal{Q}}$ provides an identification between the bosonic and the fermionic system, so that picking a subsystem on the bosonic side automatically defines a related subsystem on the fermionic side and vice versa. Our key finding is that, under identification, we have $\lambda_{\mathrm{b}}=1 / \lambda_{\mathrm{f}}$ and $J_{\mathrm{b}}=-J_{\mathrm{f}}^{-1}$, where we use $\mathrm{b}$ and $\mathrm{f}$ to refer to the bosonic and fermionic structure, respectively.

Applying our results to examples, we also discuss consequences of the derived duality for the entanglement entropy in Gaussian states related by SUSY. Though not always, entanglement entropy often turns out to be a sufficient measure (among others) of the entanglement information encoded in a quantum state [57-59]. In fact, in a number of strongly correlated systems, this quantity serves as a smoking gun to identify topological quantum order in the ground states. Examples include Kitaev's celebrated model of Majorana fermions on a honeycomb lattice [60]. In earlier works [11], the bosonic SUSY analog of this model has been realized and shown to inherit the topological properties from its fermionic partner. We will also regard this model here, as one of our examples to illustrate the aspects of entanglement dualities considering the SUSY-related Gaussian states.

Generally speaking, for noncritical ground states in $d$ dimensions (for both fermionic and bosonic systems), the entanglement entropy of a subsystem $A$ obeys the so-called "area law" (for a review, see Refs. [58,61] and references therein)

$$
\mathcal{S}(A) \propto L^{d-1}+\ldots,
$$

meaning that, in the thermodynamic limit, the leading order contribution to the entanglement entropy of $A$ with the rest of the system scales with its surface area $L^{d-1}$ when $L$ denotes the linear dimension of $A$. For critical states, however, the ellipses in Eq. (2) can contain sublinear corrections (e.g.,logarithmic corrections for free fermions), and for topologically ordered states, a universal constant called "topological entanglement entropy."

The identification provided by the supercharge $\hat{\mathcal{Q}}$ facilitates a natural connection between a subsystem in one lattice and a subsystem in the superpartner lattice. A priori, this identification does not warrant a local subsystem in one system to get mapped to a localized subsystem in its superpartner system. However, we will show that, even when well-localized subregions are identified of both lattices, the scaling of the entanglement entropy of the dual supersymmetric subsystem can be very different-on the bosonic side, it can drastically exceed the area law exhibited by the original fermionic subsystem.

In summary, this paper extends the concept of SUSY beyond a spectral mapping between (supersymmetric) quadratic Hamiltonians to discuss the general identification of fermionic and bosonic supersymmetric Gaussian systems, their subsystems, and entanglement spectra as implied by the supercharge operator. Exemplifying lattice models in one dimensions (1D) and two dimensions (2D), we investigate the locality properties of these identification maps and their consequences in the context of entanglement area laws. In doing so, we employ the idea of Kähler structure, which brings the bosonic and fermionic Gaussian states within a unified frame to work in. A further merit of this approach lies in treating the involved geometric structures independent of their matrix representation in a given basis, as discussed at length, e.g.,in Refs. [62-64].

This paper is structured as follows: In Sec. II, we review the unified Kähler structure formalism to describe bosonic and fermionic Gaussian states and apply it to supersymmetric quadratic Hamiltonians, where a charge operator induces an identification map at the classical phase space level. In Sec. III, we explore how the entanglement entropies in the bosonic and fermionic systems are related and introduce a general theorem on their entanglement spectra. In Sec. IV, we summarize our key findings complemented by lattice models as applications and discuss future work.

\section{GAUSSIAN STATES AND SUSY}

In this section, we review the unified formalism that treats both bosonic and fermionic Gaussian states on the same footing. For this, we present a hands-on introduction to the formalism of Ref. [63], which can be consulted for a more rigorous exposition. Other reviews of Gaussian states include Ref. [15].

\section{A. Bosonic and fermionic Gaussian states}

We consider a bosonic or fermionic system with $N$ degrees of freedom described by a Hilbert space $\mathcal{H}$. We can always find a basis of creation and annihilation operators which we denote as $\hat{b}_{i}$ and $\hat{b}_{i}^{\dagger}$ for bosons and as $\hat{c}_{i}$ and $\hat{c}_{i}^{\dagger}$ for fermions, but we use $\hat{a}_{i}$ and $\hat{a}_{i}^{\dagger}$ in expressions valid for both bosons and fermions (see Table I). These operators satisfy the canonical commutation or anticommutation relations (CCR/CAR)

$$
\begin{array}{ll}
{\left[\hat{b}_{i}, \hat{b}_{j}^{\dagger}\right]=\delta_{i j}} & \text { (bosons), } \\
\left\{\hat{c}_{i}, \hat{c}_{j}^{\dagger}\right\}=\delta_{i j} & \text { (fermions). }
\end{array}
$$

Out of these, we can construct a set of $2 N$ Hermitian operators

$$
\begin{array}{ll}
\hat{q}_{i}=\frac{1}{\sqrt{2}}\left(\hat{b}_{i}^{\dagger}+\hat{b}_{i}\right), \quad \hat{p}_{i}=\frac{\mathrm{i}}{\sqrt{2}}\left(\hat{b}_{i}^{\dagger}-\hat{b}_{i}\right) \quad \text { (bosons), } \\
\hat{\gamma}_{i}=\frac{1}{\sqrt{2}}\left(\hat{c}_{i}^{\dagger}+\hat{c}_{i}\right), \quad \hat{\eta}_{i}=\frac{\mathrm{i}}{\sqrt{2}}\left(\hat{c}_{i}^{\dagger}-\hat{c}_{i}\right) \quad \text { (fermions), }
\end{array}
$$

which satisfy the commutation or anticommutation relations

$$
\begin{aligned}
& {\left[\hat{q}_{i}, \hat{q}_{j}\right]=\left[\hat{p}_{i}, \hat{p}_{j}\right]=0, \quad\left[\hat{q}_{i}, \hat{p}_{j}\right]=\mathrm{i} \delta_{i j} \quad \text { (bosons), }} \\
& \left\{\hat{\gamma}_{i}, \hat{\gamma}_{j}\right\}=\left\{\hat{\eta}_{i}, \hat{\eta}_{j}\right\}=\delta_{i j}, \quad\left\{\hat{\gamma}_{i}, \hat{\eta}_{j}\right\}=0 \quad \text { (fermions). }
\end{aligned}
$$

For bosons, these operators are commonly called quadrature operators (generalized positions and momenta), while for fermions, they are called the Majorana operators.

Up to normalization, there is a unique state $|0\rangle \in \mathcal{H}$, such that $\hat{a}_{i}|0\rangle=0 \forall i$, which is called the vacuum state with respect to our choice of operators. An orthonormal basis of $\mathcal{H}$ can then be constructed by successively applying creation operators on $|0\rangle$,

$$
\left|n_{1}, \ldots, n_{N}\right\rangle=\prod_{i=1}^{N} \frac{\left(\hat{a}_{i}^{\dagger}\right)^{n_{i}}}{\sqrt{n_{i} !}}|0\rangle,
$$

where $n_{i} \in \mathbb{N}$ for bosons, and $n_{i}=0,1$ for fermions. 
TABLE I. Overview of notations for operator bases. Listed are real (self-adjoint) and complex operator bases for bosons and fermions, as well as a unified notation used throughout this paper. For an $N$-mode quantum system, indices are in the range $j, k \in\{1, \ldots, N\}$. The creation and annihilation operators, in a complex basis, satisfy (CCR/CAR).

\begin{tabular}{llr}
\hline \hline & \multicolumn{1}{c}{ Real basis } & Complex basis \\
\hline Bosons & Quadrature operators $\hat{\xi}_{\mathrm{b}} \equiv\left(\hat{q}_{j}, \hat{p}_{k}\right)$ & CCR operators $\left(\hat{b}_{j}, \hat{b}_{k}^{\dagger}\right)$ \\
Fermions & Majorana operators $\hat{\xi}_{\mathrm{f}} \equiv\left(\hat{\gamma}_{j}, \hat{\eta}_{k}\right)$ & CAR operators $\left(\hat{c}_{j}, \hat{c}_{k}^{\dagger}\right)$ \\
Unified & Hermitian operators $\hat{\xi}$ & Ladder operators $\left(\hat{a}_{j}, \hat{a}_{k}^{\dagger}\right)$ \\
\hline \hline
\end{tabular}

We can now collect the $2 \mathrm{~N}$ operators to form the vector

$$
\hat{\xi}^{a} \equiv\left\{\begin{array}{ll}
\left(\hat{q}_{1}, \ldots, \hat{q}_{N}, \hat{p}_{1}, \ldots, \hat{p}_{N}\right) & \text { (bosons) } \\
\left(\hat{\gamma}_{1}, \ldots, \hat{\gamma}_{N}, \hat{\eta}_{1}, \ldots, \hat{\eta}_{N}\right) & \text { (fermions) }
\end{array},\right.
$$

where we have the index $a=1, \ldots, 2 N$ (later, we use Latin indices exclusively for bosons and Greek indices for fermions, but for now, we use Latin indices for both). It is well known that, for both bosons and fermions, any operator $\mathcal{O}$ can be described as a power series in $\hat{\xi}^{a}$ or as a limit of such a series. For many physically relevant operators, this series will be finite and of low order. The CCR or CAR in terms of $\hat{\xi}^{a}$ read

$$
\begin{aligned}
& {\left[\hat{\xi}^{a}, \hat{\xi}^{b}\right]=\mathrm{i} \Omega^{a b} \quad \text { (bosons), }} \\
& \left\{\hat{\xi}^{a}, \hat{\xi}^{b}\right\}=G^{a b} \quad \text { (fermions), }
\end{aligned}
$$

where $\Omega^{a b}$ is called the symplectic form, and $G^{a b}$ is a metric. With respect to our choice of basis in Eq. (7), they are represented by the matrices

$$
\Omega \equiv\left(\begin{array}{cc}
0 & \mathbb{1} \\
-\mathbb{1} & 0
\end{array}\right) \quad \text { and } \quad G \equiv\left(\begin{array}{ll}
\mathbb{1} & 0 \\
0 & \mathbb{1}
\end{array}\right)
$$

and will play an important role in later formulas.

We define ${ }^{1}$ a Gaussian state $|J\rangle \in \mathcal{H}$ as the solution of ${ }^{2}$

$$
\frac{1}{2}\left(\delta^{a}{ }_{b}+\mathrm{i} J^{a}{ }_{b}\right) \hat{\xi}^{b}|J\rangle=0 .
$$

As shown in Ref. [63], a solution of Eq. (10) exists only if $J^{2}=-\mathbb{1}$ and the following compatibility conditions are satisfied:

(1) For bosons, $G^{a b}:=-J^{a}{ }_{c} \Omega^{c b}$ is a metric, i.e.,symmetric and positive definite.

(2) For fermions, $\Omega^{a b}:=J^{a}{ }_{c} G^{c b}$ is a symplectic form, i.e., antisymmetric and nondegenerate.

The matrix $J$ is called a linear complex structure.

In Eq. (10) and the rest of this paper, we use Einstein's summation convention, ${ }^{3}$ where a sum is implied over repeated

\footnotetext{
${ }^{1}$ Here, we restrict to Gaussian states with $\left\langle J\left|\hat{\xi}^{a}\right| J\right\rangle=0$, i.e., the 1point correlation function vanishes. However, the formalism extends to also include displacements $z^{a}=\left\langle J\left|\hat{\xi}^{a}\right| J\right\rangle$ for bosons, as explained in Ref. [63].

${ }^{2}$ Note that Eq. (10) only fixes $|J\rangle$ up to a complex phase. This does not cause any problems when considering individual Gaussian states, where the complex phase is unphysical. However, if considering superpositions of Gaussian states $|J\rangle+|\tilde{J}\rangle$, we would need to parametrize explicitly how the respective complex phases are related.

${ }^{3}$ All our equations with indices are fully basis independent and compatible with Penrose's abstract index notation [65]. In fact, we can even use complex bases, such as $\hat{\xi}^{a} \equiv\left(\hat{a}_{1}, \ldots, \hat{a}_{N}, \hat{a}_{1}^{\dagger}, \ldots, \hat{a}_{N}^{\dagger}\right)$ (see Ref. [63]).
}

indices (index contraction). The position of the index indicates if it can be contracted with vectors $v^{a} \in V$ in phase space or dual vectors $w_{a} \in V^{*}$ in dual phase space. Objects with two indices are often written as matrices, where matrix multiplication is the same as contraction over adjected indices. This may require a transpose, e.g., $\Omega^{a c} J^{b}{ }_{c}$ needs to be written as $\left(\Omega J^{\top}\right)^{a b}=\Omega^{a c}\left(J^{\top}\right)_{c}^{b}$ to make the indices $c$ adjacent.

The above relations introduce for every Gaussian state $|J\rangle$ the object $G^{a b}$ for bosons and $\Omega^{a b}$ for fermions, such that we have in both cases a so-called Kähler structure: This is a triplet $(G, \Omega, J)$ such that

$$
G^{a b}=-J^{a}{ }_{c} \Omega^{c b} \Leftrightarrow \Omega^{a b}=J^{a}{ }_{c} G^{c b},
$$

the equivalence following from $J^{2}=-\mathbb{1}$. Moreover, we have $J \Omega J^{\top}=\Omega$ and $J G J^{\top}=G$.

This definition of Gaussian states, unifying bosons and fermions, may appear surprising to readers more familiar with the definition of Gaussian states in terms of covariance matrices or Bogoliubov transformations. However, as shown in Ref. [63], these definitions are fully equivalent, as we review in the following.

\section{Covariance matrix}

The covariance matrix of a quantum state $|\psi\rangle$ with $\left\langle\psi\left|\hat{\xi}^{a}\right| \psi\right\rangle=0$ is defined as ${ }^{4}$

$$
\Gamma^{a b}=\left\{\begin{array}{ll}
\left\langle\psi\left|\hat{\xi}^{a} \hat{\xi}^{b}+\hat{\xi}^{b} \hat{\xi}^{a}\right| \psi\right\rangle & \text { (bosons) } \\
-\mathrm{i}\left\langle\psi\left|\hat{\xi}^{a} \hat{\xi}^{b}-\hat{\xi}^{b} \hat{\xi}^{a}\right| \psi\right\rangle & \text { (fermions) }
\end{array},\right.
$$

i.e.,the covariance matrix is exactly the expression that is not already fixed by the CCR or CAR. Given a Gaussian state $|J\rangle$ with associated Kähler structures $(G, \Omega, J)$, it follows from Eq. (10) that we have the 2-point function

$$
C_{2}^{a b}:=\left\langle J\left|\hat{\xi}^{a} \hat{\xi}^{b}\right| J\right\rangle=\frac{1}{2}\left(G^{a b}+\mathrm{i} \Omega^{a b}\right),
$$

To prove this, we define $\hat{\xi}_{ \pm}^{a}=\frac{1}{2}\left(\delta^{a}{ }_{b} \mp \mathrm{i} J^{a}{ }_{b}\right) \hat{\xi}^{b}$, which depends on $J$. With this, we find $\hat{\xi}^{a}=\hat{\xi}_{+}^{a}+\hat{\xi}_{-}^{a}$, and we have $\hat{\xi}_{-}^{a}|J\rangle=0$ and $\langle J| \hat{\xi}_{+}^{a}=0$, due to Eq. (10). This implies

$$
C_{2}^{a b}=\left\langle J\left|\hat{\xi}_{-}^{a} \hat{\xi}_{+}^{b}\right| J\right\rangle= \begin{cases}\left\langle J\left|\left[\hat{\xi}_{-}^{a}, \hat{\xi}_{+}^{b}\right]\right| J\right\rangle & \text { (bosons) } \\ \left\langle J\left|\left\{\hat{\xi}_{-}^{a}, \hat{\xi}_{+}^{b}\right\}\right| J\right\rangle & \text { (fermions) }\end{cases}
$$

\footnotetext{
${ }^{4}$ Some authors use a different normalization or sign. The extension to states with $\left\langle\psi\left|\hat{\xi}^{a}\right| \psi\right\rangle \neq 0$ is also straightforward and explained in Ref. [63].
} 
due to $\left\langle J\left|\hat{\xi}_{+}^{b} \hat{\xi}_{-}^{a}\right| J\right\rangle=0$. Finally, the commutator or anticommutator above can be evaluated using Eq. (8) to be

$$
\begin{array}{ll}
{\left[\hat{\xi}_{-}^{a}, \hat{\xi}_{+}^{b}\right]=\frac{1}{4}(\mathbb{1}+\mathrm{i} J)^{a}{ }_{c} \mathrm{i} \Omega^{c d}(\mathbb{1}-\mathrm{i} J)^{b}{ }_{d}} & \text { (bosons), } \\
\left\{\hat{\xi}_{-}^{a}, \hat{\xi}_{+}^{b}\right\}=\frac{1}{4}(\mathbb{1}+\mathrm{i} J)^{a}{ }_{c} G^{c d}(\mathbb{1}-\mathrm{i} J)^{b}{ }_{d} & \text { (fermions), }
\end{array}
$$

which in both cases combines to $\frac{1}{2}(G+\mathrm{i} \Omega)$ via Eq. (11).

We can reverse this argument to use $C_{2}^{a b}$ (and thus the covariance matrix $\Gamma^{a b}$ contained in it) of a general state $|\psi\rangle$, with $\left\langle\psi\left|\hat{\xi}^{a}\right| \psi\right\rangle=0$, to check if $|\psi\rangle$ is a Gaussian state, i.e., $|\psi\rangle=|J\rangle$ and find $J$. For this, we first compute $G^{a b}=$ $2 \operatorname{Re}\left\langle\psi\left|\hat{\xi}^{a} \hat{\xi}^{b}\right| \psi\right\rangle$ and $\Omega^{a b}=2 \operatorname{Im}\left\langle\psi\left|\hat{\xi}^{a} \hat{\xi}^{b}\right| \psi\right\rangle$ and then invert Eq. (11) to compute

$$
J^{a}{ }_{b}=\Omega^{a c}\left(G^{-1}\right) c b .
$$

One can then show [63] that $J^{2}=-\mathbb{1}$ is necessary and sufficient for $|\psi\rangle$ to be the Gaussian state $|J\rangle$, i.e., a solution of Eq. (10). However, if $J^{2} \neq-\mathbb{1},|\psi\rangle$ is not a Gaussian state.

\section{Bogoliubov transformations}

These transformations map Gaussian states into Gaussian states, hence, are also termed Gaussian transformations. For a Gaussian state $|J\rangle$ annihilated by a set of annihilation operators $\hat{a}_{i}^{\prime}$, i.e., $\hat{a}_{i}^{\prime}|J\rangle=0$, the following transformation relates them to the original $\hat{a}_{i}$

$$
\hat{a}_{i}^{\prime}=\sum_{j}\left(\alpha_{i j} \hat{a}_{i}+\beta_{i j} \hat{a}_{j}^{\dagger}\right)
$$

where the matrix elements $\alpha_{i j}$ and $\beta_{i j}$ characterize the transformation. Defining a Gaussian state $\left|J_{0}\right\rangle$ as the state annihilated by all $\hat{a}_{i}$, i.e., $\hat{a}_{i}\left|J_{0}\right\rangle=0$, we can use Eqs. (16) and (9) to compute that $J_{0}$ is represented by the matrix

$$
J_{0} \equiv\left(\begin{array}{cc}
0 & \mathbb{1} \\
-\mathbb{1} & 0
\end{array}\right)
$$

from which we deduce the resulting Bogoliubov transformed state $|J\rangle$ with $J=M J_{0} M^{-1}$, where the matrix $M$ is [64]

$$
M=\left(\begin{array}{ll}
\operatorname{Re} \alpha+\operatorname{Re} \beta & \operatorname{Im} \beta-\operatorname{Im} \alpha \\
\operatorname{Im} \alpha+\operatorname{Im} \beta & \operatorname{Re} \alpha-\operatorname{Re} \beta
\end{array}\right) .
$$

The matrix $M$ is a group element of the symplectic group $\operatorname{Sp}(2 N, \mathbb{R})$ for bosons or the orthogonal group $\mathrm{O}(2 N, \mathbb{R})$ for fermions, which induces the unitary representation of Gaussian transformations on the Hilbert space [63].

Example 1. The simplest bosonic Gaussian state is the ground state of the harmonic oscillator with Hamiltonian $\hat{H}=$ $\frac{1}{2}\left(\hat{p}^{2}+\omega^{2} \hat{q}^{2}\right)$ that takes the form

$$
|J\rangle=\frac{1}{\cosh \frac{\rho}{2}} \sum_{n=0}^{\infty}\left(-\tanh \frac{\rho}{2}\right)^{n}|2 n\rangle,
$$

with respect to the basis in Eq. (6) and $\rho=\log \omega$. Its covariance matrix $\Gamma^{a b}=G^{a b}$ and complex structure as

$$
\Gamma=G \equiv\left(\begin{array}{cc}
\omega & 0 \\
0 & \frac{1}{\omega}
\end{array}\right) \text { and } J \equiv\left(\begin{array}{cc}
0 & \omega \\
-\frac{1}{\omega} & 0
\end{array}\right),
$$

with respect to the basis $\hat{\xi}^{a} \equiv(\hat{q}, \hat{p})$.

The simplest fermionic Gaussian states are the basis states $\left|J_{+}\right\rangle=|0\rangle$ and $\left|J_{-}\right\rangle=|1\rangle$, which are also the only Gaussian states for a single degree of freedom. Their covariance matrices $\Gamma_{ \pm}=\Omega_{ \pm}$and complex structures $J_{ \pm}$happen to coincide in the basis $\hat{\xi}^{a} \equiv(\hat{q}, \hat{p})$ as

$$
\Gamma_{ \pm}=\Omega_{ \pm} \equiv J_{ \pm} \equiv\left(\begin{array}{cc}
0 & \pm 1 \\
\mp 1 & 0
\end{array}\right) .
$$

In summary, this section has reviewed how bosonic and fermionic Gaussian states can be efficiently described in a unified formalism using the triplet $(G, \Omega, J)$ of Kähler structures. Physical properties, such as expectation values or entanglement entropies, can be directly computed from them.

\section{B. Supercharge operator and supersymmetric Gaussian states}

We will now consider a system that contains both bosonic and fermionic degrees of freedom. We denote the bosonic operators by $\hat{\xi}_{\mathrm{b}}^{a}$ and the fermionic ones by $\hat{\xi}_{\mathrm{f}}^{\alpha}$, where we use Latin letters for bosons and Greek letters for fermions. The commutation and anticommutation relations then read

$$
\left[\hat{\xi}_{\mathrm{b}}^{a}, \hat{\xi}_{\mathrm{b}}^{b}\right]=\mathrm{i} \Omega_{\mathrm{b}}^{a b} \quad \text { and } \quad\left\{\hat{\xi}_{\mathrm{f}}^{\alpha}, \hat{\xi}_{\mathrm{f}}^{\beta}\right\}=G_{\mathrm{f}}^{\alpha \beta},
$$

while the bosonic and the fermionic operators commute $\left[\hat{\xi}_{\mathrm{b}}^{a}, \hat{\xi}_{\mathrm{f}}^{\alpha}\right]=0$.

The SUSY transformation between the bosonic and the fermionic degrees of freedom can be generated by a Hermitian supercharge operator [10]

$$
\hat{\mathcal{Q}}=R_{\alpha a} \hat{\xi}_{\mathrm{f}}^{\alpha} \hat{\xi}_{\mathrm{b}}^{a},
$$

with a real-valued $R$. As mentioned already in Eq. (1), this supercharge defines a supersymmetric Hamiltonian

$\hat{H}=\frac{1}{2}\{\hat{\mathcal{Q}}, \hat{\mathcal{Q}}\}=\frac{1}{2} h_{a b}^{\mathrm{b}} \hat{\xi}_{\mathrm{b}}^{a} \hat{\xi}_{\mathrm{b}}^{b}+\frac{\mathrm{i}}{2} h_{\alpha \beta}^{\mathrm{f}} \hat{\xi}_{\mathrm{f}}^{\alpha} \hat{\xi}_{\mathrm{f}}^{\beta} \equiv \hat{H}_{\mathrm{b}}+\hat{H}_{\mathrm{f}}$,

which splits into a bosonic part $\hat{H}_{\mathrm{b}}$ and a fermionic part $\hat{H}_{\mathrm{f}}$. Their Hamiltonian forms are

$$
\begin{aligned}
& h_{\alpha \beta}^{\mathrm{f}}=R_{\alpha a} \Omega^{a b} R_{b \beta}^{\top}, \\
& h_{a b}^{\mathrm{b}}=R_{a \alpha}^{\top} G^{\alpha \beta} R_{\beta b},
\end{aligned}
$$

which satisfy $h_{\alpha \beta}^{\mathrm{f}}=-h_{\beta \alpha}^{\mathrm{f}}$ and $h_{a b}^{\mathrm{b}}=h_{b a}^{\mathrm{b}}$. Note that the full Hamiltonian's ground state energy $E_{0}=\mathrm{i} R_{\alpha a} R_{\beta b} G^{\beta \alpha} \Omega^{b a}=$ $\mathrm{i} \operatorname{tr}\left(G R \Omega^{\top} R^{\top}\right)=0$ vanishes, as the bosonic and the fermionic contributions cancel each other.

The excitation spectrum of $\hat{H}_{\mathrm{b}}$ and $\hat{H}_{\mathrm{f}}$ can be derived by diagonalizing the Lie generators $K_{\mathrm{b}}$ and $K_{\mathrm{f}}$, defined via the relations ${ }^{5}$

$$
\left[\hat{H}, \hat{\xi}_{\mathrm{b}}^{a}\right]=\left(K_{\mathrm{b}}\right)^{a}{ }_{b} \hat{\xi}_{\mathrm{b}}^{b} \quad \text { and } \quad\left[\hat{H}, \hat{\xi}_{\mathrm{f}}^{\alpha}\right]=\left(K_{\mathrm{f}}\right)^{\alpha}{ }_{\beta} \hat{\xi}_{\mathrm{f}}^{\beta} .
$$

One can show [63] that these matrices are Lie algebra elements satisfying

$$
K_{\mathrm{b}} \Omega=-\Omega K_{\mathrm{b}}^{\top} \quad \text { and } \quad K_{\mathrm{f}} G=-G K_{\mathrm{f}}^{\top},
$$

which implies $K_{\mathrm{b}} \in \mathfrak{s p}(2 N, \mathbb{R})$ and $K_{\mathrm{f}} \in \mathfrak{s o}(2 N, \mathbb{R})$. Using the relations in Eq. (3) allows us to compute them explicitly as

$$
\begin{gathered}
\left(K_{\mathrm{b}}\right)^{a}{ }_{b}=\frac{1}{2} \Omega^{a c}\left(h_{c b}+h_{b c}\right)=\Omega^{a c} R_{c \alpha}^{\top} G^{\alpha \beta} R_{\beta b}, \\
\left(K_{\mathrm{f}}\right)^{\alpha}{ }_{\beta}=\frac{1}{2} G^{\alpha \gamma}\left(h_{\gamma \beta}-h_{\beta \gamma}\right)=G^{\alpha \gamma} R_{\gamma a} \Omega^{a b} R_{b \beta}^{\top} .
\end{gathered}
$$

\footnotetext{
${ }^{5}$ Alternatively, one can also exploit the Heisenberg equation of motion leading to $\frac{d}{d t} \hat{\xi}_{\mathrm{b}}^{a}=\mathrm{i}\left[\hat{H}, \hat{\xi}_{\mathrm{b}}^{a}\right]=\mathrm{i}\left(K_{\mathrm{b}}\right)^{a}{ }_{b} \hat{\xi}_{\mathrm{b}}^{b}$ and similarly for $\hat{\xi}_{\mathrm{f}}^{\alpha}$.
} 
From this, it is evident that $K_{\mathrm{b}}$ and $K_{\mathrm{f}}$ are isospectral except for the degeneracy of potential zero eigenvalues.

The ground state of $\hat{H}$ is given by the tensor product

$$
|\mathrm{GS}\rangle=\left|J_{\mathrm{b}}\right\rangle \otimes\left|J_{\mathrm{f}}\right\rangle,
$$

where the associated $J_{\mathrm{b}}$ and $J_{\mathrm{f}}$ are computed from the generators as $[63,66]^{6}$

$$
J_{\mathrm{b}}=\left|K_{\mathrm{b}}^{-1}\right| K_{\mathrm{b}} \quad \text { and } \quad J_{\mathrm{f}}=\left|K_{\mathrm{f}}^{-1}\right| K_{\mathrm{f}} .
$$

These formulas may be surprising at first sight, but they can be readily checked using a basis, where the individual normal modes of $\hat{H}$ decouple. In this basis, we have

$$
\hat{H}=\sum_{i} \frac{\omega_{i}}{2}\left(\hat{n}_{i}^{\mathrm{b}}+\hat{n}_{i}^{\mathrm{f}}\right),
$$

where $\hat{n}_{i}=\hat{a}_{i}^{\dagger} \hat{a}_{i}$ are the normal mode number operators and $\omega_{i}$ are the one-particle excitation energies. Note that, due to $h_{a b}^{\mathrm{b}}$ being positive, all $\omega_{i}$ are positive, and we choose $\hat{n}_{i}^{\mathrm{f}}$, such that excitations increase energy. If we go into the associated basis $\hat{\xi}^{a}$, where $\hat{n}_{i}^{\mathrm{b}}=\frac{1}{2}\left[\left(\hat{q}_{i}^{\mathrm{b}}\right)^{2}+\left(\hat{p}_{i}^{\mathrm{b}}\right)^{2}\right]$ and $\hat{n}_{i}^{\mathrm{f}}=\mathrm{i} \hat{\gamma}_{i} \hat{\eta}_{i}$, the matrix representations of the generators are

$$
K_{\mathrm{b}} \equiv K_{\mathrm{f}} \equiv \oplus_{i}\left(\begin{array}{cc}
0 & \omega_{i} \\
-\omega_{i} & 0
\end{array}\right) .
$$

In this specific basis, $J_{\mathrm{b}}$ and $J_{\mathrm{f}}$ assume the standard form from Eq. (18), which then implies Eq. (33).

Example 2. The simplest supersymmetric Hamiltonian consists of one bosonic and one fermionic degree of freedom. The respective supercharge operator is given by

$$
\hat{\mathcal{Q}}=\hat{q} \hat{\gamma}+\hat{p} \hat{\eta}
$$

for which we find the Hamiltonian

$$
\hat{H}=\hat{\mathcal{Q}}^{2}=\frac{1}{2}\left(\hat{q}^{2}+\hat{p}^{2}\right)+\frac{\mathrm{i}}{2}(\hat{\gamma} \hat{\eta}-\hat{\eta} \hat{\gamma}) .
$$

(equivalent forms of $\hat{\mathcal{Q}}$ and $\hat{H}$ in terms of complex bosonic and fermionic operators are shown in the introduction). The associated Lie algebra generators are then given by

$$
K_{\mathrm{b}} \equiv K_{\mathrm{f}} \equiv\left(\begin{array}{cc}
0 & 1 \\
-1 & 0
\end{array}\right),
$$

and the associated ground state is $|\mathrm{GS}\rangle=\left|0_{\mathrm{b}}\right\rangle \otimes\left|0_{\mathrm{f}}\right\rangle$.

\section{Supersymmetric identification maps}

We introduced supersymmetric Hamiltonians through the supercharge operator $\hat{\mathcal{Q}}$ as $\hat{H}=\hat{H}_{\mathrm{b}}+\hat{H}_{\mathrm{f}}$, where $\hat{H}_{\mathrm{b}}$ and $\hat{H}_{\mathrm{f}}$ have identical one-particle spectra. Both the bosonic and the fermionic part are described classically by phase spaces $V_{\mathrm{b}} \simeq \mathbb{R}^{2 N}$ and $V_{\mathrm{f}} \simeq \mathbb{R}^{2 N}$ (with the corresponding dual spaces denoted by $V_{\mathrm{b}}^{*}$ and $V_{\mathrm{f}}^{*}$ ), respectively, such that $V_{\mathrm{b}}$ is equipped with the symplectic form $\Omega_{\mathrm{b}}$, and $V_{\mathrm{f}}$ is equipped with a metric $G_{\mathrm{f}}$. The respective other structure in each space, i.e.,a metric

\footnotetext{
${ }^{6}$ Note that applying a function $f(K)$, such as the absolute value, to a diagonalizable matrix $K=U^{-1} D U$, where $D$ is a diagonal matrix containing the eigenvalues of $K$, is equivalent to applying $f$ to its eigenvalues, i.e., $f(K)=U^{-1} f(D) U$.
}

$G$ on $V_{\mathrm{b}}$ and a symplectic form $\Omega$ on $V_{\mathrm{f}}$, is defined by the ground state $|J\rangle=\left|J_{\mathrm{b}}\right\rangle \otimes\left|J_{\mathrm{f}}\right\rangle$ of $\hat{H}$.

In this section, we now use the supercharge $\hat{\mathcal{Q}}$ to construct linear maps between the two phase spaces $L_{1}: V_{\mathrm{b}} \rightarrow V_{\mathrm{f}}$ and $L_{2}: V_{\mathrm{f}} \rightarrow V_{\mathrm{b}}$ that identify the spaces in such a way that the symplectic forms and metrics are mapped onto each other.

Under the above assumption that $R_{a \alpha}$ is real, the supercharge operator $\hat{\mathcal{Q}}=R_{\alpha a} \hat{\xi}_{\mathrm{f}}^{\alpha} \hat{\xi}_{\mathrm{b}}^{a}$ induces the supersymmetric identification maps $T_{1}: V_{\mathrm{b}} \rightarrow V_{\mathrm{f}}$ and $T_{2}: V_{\mathrm{f}} \rightarrow V_{\mathrm{b}}$ as

$$
\left(T_{1}\right)^{\alpha}{ }_{a}=G^{\alpha \beta} R_{\beta a} \text { and }\left(T_{2}\right)^{a}{ }_{\alpha}=\Omega^{a b} R_{b \alpha}^{\top} .
$$

These are related to the Lie generators noting $K_{\mathrm{b}}=T_{2} T_{1}$ and $K_{\mathrm{f}}=T_{1} T_{2}$. Hence, $T_{2}$ maps the eigenvectors of $K_{\mathrm{f}}$ (in $V_{\mathrm{f}, \mathbb{C}}$, the complexification on $V_{\mathrm{f}}$ ) to the eigenvectors of $K_{\mathrm{b}}$ with the same eigenvalue, and for $T_{1}$, the analogous holds:

$$
\begin{aligned}
& K_{\mathrm{b}} v_{\mathrm{b}}= \pm \mathrm{i} \lambda_{v} v_{\mathrm{b}} \Rightarrow K_{\mathrm{f}} T_{1} v_{\mathrm{b}}= \pm \mathrm{i} \lambda_{v} T_{1} v_{\mathrm{b}}, \\
& K_{\mathrm{f}} w_{\mathrm{f}}= \pm \mathrm{i} \lambda_{w} w_{\mathrm{f}} \Rightarrow K_{\mathrm{b}} T_{2} w_{\mathrm{f}}= \pm \mathrm{i} \lambda_{w} T_{2} w_{\mathrm{f}} .
\end{aligned}
$$

If only the spaces $V_{\mathrm{b}}$ and $V_{\mathrm{f}}$ are given, each equipped with Kähler structures $(G, \Omega, J)$, then there exists a large class of potential identification maps; ${ }^{7}$ however, the choice of $R_{\alpha b}$ fixes this freedom.

We can use the supersymmetric identification maps to construct normalized identification maps $L_{1}: V_{\mathrm{b}} \rightarrow V_{\mathrm{f}}$ and $L_{2}: V_{\mathrm{f}} \rightarrow V_{\mathrm{b}}$ as

$$
L_{1}=\left|K_{\mathrm{f}}^{-1}\right|^{1 / 2} T_{1}, \quad L_{2}=\left|K_{\mathrm{b}}^{-1}\right|^{1 / 2} T_{2},
$$

(where the form of $L_{1}$ was identified in Ref. [11]). These have the property that their products exactly reproduce the linear complex structures

$$
L_{1} L_{2}=J_{\mathrm{f}} \quad \text { and } \quad L_{2} L_{1}=J_{\mathrm{b}},
$$

of the ground state of $\hat{H}$.

To see this, it is convenient to work in the eigenbases of the generators $K_{\mathrm{b}}$ and $K_{\mathrm{f}}$. Let $v^{( \pm k)} \in V_{\mathrm{b}, \mathbb{C}}$ denote a basis of eigenvectors of $K_{\mathrm{b}}$ with eigenvalues $\pm \mathrm{i} \lambda_{k}$. Then $\left\{T_{1} v^{( \pm k)}\right\}$ is a basis of $V_{\mathrm{f}, \mathbb{C}}$ diagonalizing $K_{\mathrm{f}}$. In fact, with respect to these bases, $K_{\mathrm{b}}$ and $K_{\mathrm{f}}$ are represented by the same matrix. Accordingly, also $\left|K_{\mathrm{f}}^{-1}\right|^{1 / 2}$ and $\left|K_{\mathrm{b}}^{-1}\right|^{1 / 2}$ are represented by the same matrices. From this follows, in particular,

$$
\begin{aligned}
& L_{1}=\left|K_{\mathrm{f}}^{-1}\right|^{1 / 2} T_{1}=T_{1}\left|K_{\mathrm{b}}^{-1}\right|^{1 / 2}, \\
& L_{2}=\left|K_{\mathrm{b}}^{-1}\right|^{1 / 2} T_{2}=T_{2}\left|K_{\mathrm{f}}^{-1}\right|^{1 / 2},
\end{aligned}
$$

\footnotetext{
${ }^{7}$ Given an identification map $T_{1}: V_{\mathrm{b}} \rightarrow V_{\mathrm{f}}$, we can define a new identification $T_{1}^{\prime}=U_{\mathrm{f}} T_{1} U_{\mathrm{b}}$, where both $U_{\mathrm{b}}: V_{\mathrm{b}} \rightarrow V_{\mathrm{b}}$ and $U_{\mathrm{f}}: V_{\mathrm{f}} \rightarrow$ $V_{\mathrm{f}}$ need to preserve the respective Kähler structures. This implies that $U_{\mathrm{b}}$ and $U_{\mathrm{f}}$ form a representation of the group $U(N)$. In our case, we also would like that $T_{1}$ maps $K_{\mathrm{b}}$ onto $K_{\mathrm{f}}$, which implies that the respective symmetry group will depend on the degeneracy of the one-particle spectrum. If $K_{\mathrm{b}}$ (and thus also $K_{\mathrm{f}}$ ) has $m$ distinct eigenvalue pairs $\pm \mathrm{i} \lambda_{i}$ with degeneracy $d_{i}$ such that $\sum_{i=1}^{m} d_{i}=N$, the resulting symmetry group will be $U\left(d_{1}\right) \times \cdots \times U\left(d_{m}\right)$. Only if the Hamiltonian is fully degenerate with $N$ eigenvalue pairs $\pm \lambda$, this will lead to the maximal symmetry group $U(N)$ of possible identification maps $T_{1}^{\prime}$.
} 
and hence, we have

$$
\begin{aligned}
L_{1} L_{2} & =\left|K_{\mathrm{f}}^{-1}\right|^{1 / 2} T_{1}\left|K_{\mathrm{b}}^{-1}\right|^{1 / 2} T_{2} \\
& =\left|K_{\mathrm{f}}^{-1}\right| T_{1} T_{2}=\left|K_{\mathrm{f}}^{-1}\right| K_{\mathrm{f}}=J_{\mathrm{f}}, \\
L_{2} L_{1} & =\left|K_{\mathrm{b}}^{-1}\right|^{1 / 2} T_{2}\left|K_{\mathrm{f}}^{-1}\right|^{1 / 2} T_{1} \\
& =\left|K_{\mathrm{b}}^{-1}\right| T_{2} T_{1}=\left|K_{\mathrm{b}}^{-1}\right| K_{\mathrm{b}}=J_{\mathrm{b}} .
\end{aligned}
$$

In the following, we use the identification maps to associate both linear observables and quadratic forms between the two supersymmetric partner systems. For this, it is important to note that, since the identification maps and their inverses act on the phase spaces, i.e.,they act on upper indices from the left, their corresponding transposes act on the dual phase spaces, i.e.,on lower indices, as

$$
\begin{aligned}
& V_{\mathrm{b}} \stackrel{L_{1}}{\rightarrow} V_{\mathrm{f}}, \quad V_{\mathrm{f}} \stackrel{\left(L_{1}\right)^{-1}}{\longrightarrow} V_{\mathrm{b}}, \quad V_{\mathrm{b}}^{*} \stackrel{\left(L_{1}^{\top}\right)^{-1}}{\longrightarrow} V_{\mathrm{f}}^{*}, \quad V_{\mathrm{f}}^{*} \stackrel{L_{1}^{\top}}{\longrightarrow} V_{\mathrm{b}}^{*}, \\
& V_{\mathrm{f}} \stackrel{L_{2}}{\longrightarrow} V_{\mathrm{b}}, \quad V_{\mathrm{b}} \stackrel{\left(L_{2}\right)^{-1}}{\longrightarrow} V_{\mathrm{f}}, \quad V_{\mathrm{f}}^{*} \stackrel{\left(L_{2}^{\top}\right)^{-1}}{\longrightarrow} V_{\mathrm{b}}^{*}, \quad V_{\mathrm{b}}^{*} \stackrel{L_{2}^{\top}}{\longrightarrow} V_{\mathrm{f}}^{*} .
\end{aligned}
$$

For example, let $\hat{s}=s_{a} \hat{\xi}_{\mathrm{b}}^{a}$ be a linear operator on the bosonic system. Then

$$
L_{2}(\hat{s})=s_{a}\left(L_{2}\right)^{a}{ }_{\alpha} \hat{\xi}_{\mathrm{f}}^{\alpha},
$$

is the linear fermionic operator associated to it by the identification map $L_{1}$. Analogously, if $\hat{r}=r_{\alpha} \hat{\xi}_{f}^{\alpha}$ is a fermionic operator, the identification map $L_{1}$ associates the bosonic operator

$$
L_{1}(\hat{r})=r_{\alpha}\left(L_{1}\right)^{\alpha}{ }_{a} \hat{\xi}_{\mathrm{b}}^{a}
$$

with it. In this sense, the identification maps always identify corresponding pairs of eigenmodes of the SUSY Hamiltonian with each other: If we diagonalize the SUSY Hamiltonian as

$$
\hat{\mathcal{Q}}^{2}=\sum_{i} \omega_{i}\left(\hat{b}_{i}^{\dagger} \hat{b}_{i}+\hat{c}_{i}^{\dagger} \hat{c}_{i}\right)
$$

then assuming that all $\omega_{i}$ are different, we always have

$$
L_{1}\left(\hat{c}_{i}\right)=e^{\mathrm{i} \phi_{i, 1}} \hat{b}_{i}, \quad L_{2}\left(\hat{b}_{i}\right)=e^{\mathrm{i} \phi_{i, 2}} \hat{c}_{i},
$$

for all $i=1, \ldots, N$, because of Eq. (41). Also, due to Eq. (42), the complex phases are such that $e^{\mathrm{i} \phi_{i, 1}} e^{\mathrm{i} \phi_{i, 2}}=-\mathrm{i}$, since $J_{\mathrm{b}}\left(\hat{b}_{i}\right)=-\mathrm{i} \hat{b}_{i}$ and $J_{\mathrm{f}}\left(\hat{c}_{i}\right)=-\mathrm{i} \hat{c}_{i}$ as follows from Eqs. (11) and (9) (expressed in the complex bases).
Example 3. The supercharge operator $\hat{\mathcal{Q}}$ introduced in Example 2 induces the rather simple identification maps represented by the matrices

$$
L_{1} \equiv\left(\begin{array}{ll}
1 & 0 \\
0 & 1
\end{array}\right), \quad L_{2} \equiv\left(\begin{array}{cc}
0 & 1 \\
-1 & 0
\end{array}\right) .
$$

Accordingly, the Hermitian mode operators are identified as

$$
\begin{array}{ll}
L_{1}(\hat{\gamma})=\hat{q}, & L_{1}(\hat{\eta})=\hat{p}, \\
L_{2}(\hat{q})=\hat{\eta}, & L_{2}(\hat{p})=-\hat{\gamma} .
\end{array}
$$

\section{Application: supersymmetric Kitaev chain}

In this section, we choose the well-known Kitaev chain [67] of $N$ sites with open boundary conditions as a concrete application for the formalism above and investigate the physical properties of the identification maps. In our construction, the supersymmetric partner of the Kitaev chain resembles the Kane-Lubensky (KL) chain [13]. We are interested in addressing the question: To what extent do the identification maps preserve the localization properties of operators, when mapping them from one system to its SUSY partner?

The form of the fermionic Kitaev chain Hamiltonian which we study is obtained by considering a real pairing and setting its magnitude equal to the hopping $(t)$ in the original model proposed in [67]:

$$
\hat{H}_{\mathrm{f}}=\frac{\mu}{2} \sum_{i=1}^{N}\left(\hat{c}_{i}^{\dagger} \hat{c}_{i}-\hat{c}_{i} \hat{c}_{i}^{\dagger}\right)+t \sum_{i=1}^{N-1}\left(\hat{c}_{i+1}^{\dagger} \hat{c}_{i}-\hat{c}_{i+1}^{\dagger} \hat{c}_{i}^{\dagger}+\text { H.c. }\right),
$$

where $\mu$ denotes the chemical potential. A supercharge which generates this Hamiltonian as the fermionic part of $\hat{\mathcal{Q}}^{2}=$ $\hat{H}_{\mathrm{f}}+\hat{H}_{\mathrm{b}}$ is given by

$$
\begin{aligned}
\hat{\mathcal{Q}} & =\sqrt{\mu} \sum_{i=1}^{N} \hat{c}_{i} \hat{b}_{i}^{\dagger}+\frac{t}{\sqrt{\mu}} \sum_{i=1}^{N-1}\left(\hat{c}_{i} \hat{b}_{i+1}+\hat{c}_{i} \hat{b}_{i+1}^{\dagger}\right)+\text { H.c. } \\
& =\sqrt{\mu} \sum_{i=1}^{N}\left(\hat{\gamma}_{i} \hat{q}_{i}+\hat{\eta}_{i} \hat{p}_{i}\right)+\frac{2 t}{\sqrt{\mu}} \sum_{i=1}^{N-1} \hat{\gamma}_{i+1} \hat{q}_{i} .
\end{aligned}
$$

Its bosonic part resembles the KL chain, a well-studied model in topological mechanics [13]:

$$
\begin{aligned}
\hat{H}_{\mathrm{b}}= & \frac{\mu}{2} \sum_{i=1}^{N} \hat{p}_{i}^{2}+\frac{4 t^{2}+\mu^{2}}{2 \mu} \sum_{i=2}^{N} \hat{q}_{i}^{2}+\frac{\mu}{2} \hat{q}_{1}^{2}+2 t \sum_{i=1}^{N-1} \hat{q}_{i} \hat{q}_{i+1} \\
= & \frac{\mu}{2}\left(\hat{b}_{1} \hat{b}_{1}^{\dagger}+\hat{b}_{1}^{\dagger} \hat{b}_{1}\right)+\sum_{i=2}^{N}\left[\frac{\mu}{2}\left(1+\frac{2 t^{2}}{\mu^{2}}\right)\left(\hat{b}_{i}^{\dagger} \hat{b}_{i}+\hat{b}_{i} \hat{b}_{i}^{\dagger}\right)\right. \\
& \left.+t\left(\hat{b}_{i-1} \hat{b}_{i}^{\dagger}+\hat{b}_{i-1} \hat{b}_{i}\right)+\frac{t^{2}}{\mu}\left(\hat{b}_{i} \hat{b}_{i}\right)+\text { H.c. }\right] .
\end{aligned}
$$

Denoting the energy eigenmodes of the system with primed operators, the SUSY Hamiltonian can be diagonalized as

$$
\hat{\mathcal{Q}}^{2}=\hat{H}_{\mathrm{f}}+\hat{H}_{\mathrm{b}}=\sum_{i=1}^{N} \omega_{i}\left(\hat{b}_{i}^{\prime \dagger} \hat{b}_{i}^{\prime}+\hat{c}_{i}^{\prime \dagger} \hat{c}_{i}^{\prime}\right) .
$$

Figure 1 schematically shows the spectrum of the Kitaev chain, which is in a trivial phase for $|t / \mu|<1 / 2$ and in a 


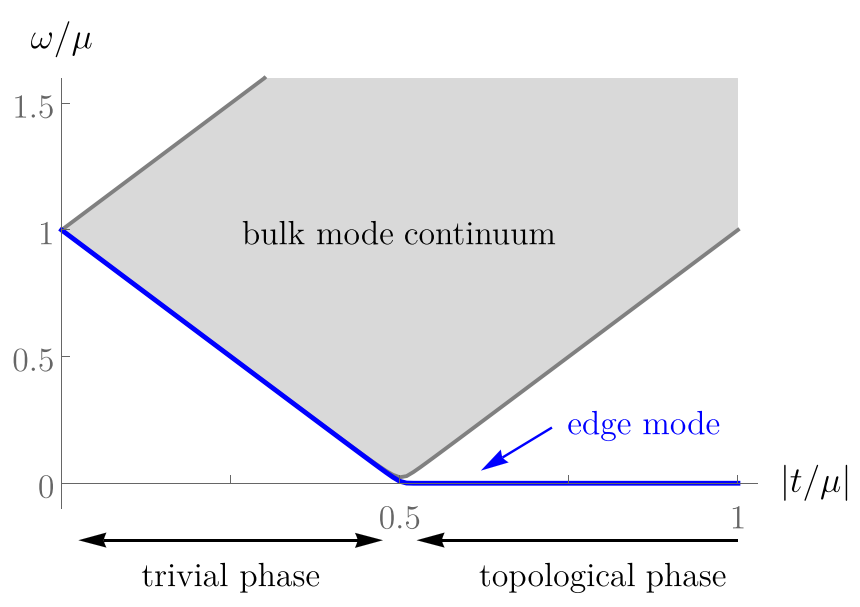

FIG. 1. Spectrum of the Kitaev chain with open ends. The system is in a trivial phase for $|t / \mu|<1 / 2$, critical at $t / \mu= \pm 1 / 2$, and topological otherwise, with edge modes appearing.

topological phase otherwise. The bulk gap closes at the critical point $t / \mu= \pm 1 / 2$ in the limit of large $N$. The trivial phase is featureless; all eigenmodes together form a bulk mode continuum. However, as the system enters the topological phase for $|t / \mu|>1 / 2$, an edge mode gradually separates from the continuum and stabilizes at zero energy (albeit with an exponentially small gap with $N$ ) as a telltale signature of the topological phase. On the fermionic side, i.e.,for the Kitaev chain, the edge modes are localized at both ends of the chain. In contrast, on the bosonic side, i.e.,for the KL chain, they are localized only at one end (here the left end) of the chain. For completeness, we mention that, in the KL chain, there exists a nonlinear zero mode (soliton) that can reverse the location of the edge mode [68]; however, that falls beyond the ambit of the present setting. The localization of the edge mode at the boundaries of the chain is exponential, in the sense that, when writing the edge mode operator as $\hat{c}_{N}^{\prime}=\sum_{j} \alpha_{j} \hat{c}_{j}+\beta_{j} \hat{c}_{j}^{\dagger}$ or
$\hat{b}_{N}^{\prime}=\sum_{j} \alpha_{j} \hat{b}_{j}+\beta_{j} \hat{b}_{j}^{\dagger}$, the quantities $\left|\alpha_{j}\right|^{2}$ and $\left|\beta_{j}\right|^{2}$ decay exponentially away from the concerned edge.

The appearance and localization of the edge modes have consequences for the properties of the identification maps. They affect to what extent the identification maps preserve the locality of the onsite observables in a system when mapping them onto its SUSY partner, as visualized in Fig. 2. From above, we know that the identification maps exactly map corresponding eigenmodes of the partner Hamiltonians to each other and that we can choose the relative phase factor such that

$$
L_{1}\left(\hat{c}_{i}^{\prime}\right)=\hat{b}_{i}^{\prime}, \quad L_{2}\left(\hat{b}_{i}^{\prime}\right)=-\mathrm{i} \hat{c}_{i}^{\prime} .
$$

Thus, at the point $(t=0)$, where the individual chain sites can be chosen as eigenmodes of the partner Hamiltonians, the identification maps exactly associate the fermionic and bosonic chain sites one to one, maintaining their ordering.

This feature of locality of the identification maps is conspicuous throughout the trivial phase, except the onsite localization at $t=0$ now transforms to an exponential one (with a length scale falling with the spectral gap), as seen in Fig. 2(a) for a chain of $N=30$ sites. In detail, in the trivial phase, the identification maps associate single site operators $\hat{c}_{i}$ and $\hat{b}_{i}$ with operators $L_{1}\left(\hat{c}_{k}\right)=\sum_{j} \alpha_{k j} \hat{b}_{j}+\beta_{k j} \hat{b}_{j}$, such that $\left|\alpha_{k j}\right|^{2}$ and $\left|\beta_{k j}\right|^{2}$ decay exponentially in $|k-j|$. Likewise, in the trivial phase, $L_{2}$ maps onsite bosonic operators to exponentially localized fermionic operators.

In the topological phase, however, the identification maps develop nonlocal features, as can be seen in Fig. 2(c). Here, a fermionic site operator $\hat{c}_{k}$ (e.g.,in the figure, $k=15$ in a chain of $N=30$ sites) when mapped to the operator $L_{1}\left(\hat{c}_{k}\right)$ on the bosonic side, can acquire a significant component located at the left edge of the bosonic chain, which is the edge where also the bosonic edge mode is localized. If we shift the original fermionic site to further right, the edge contribution to $L_{1}\left(\hat{c}_{k}\right)$ decays, and the localization of the resulting observable gains prominence. On the other hand, if we move the original site to the left, the edge contribution to $L_{1}\left(\hat{c}_{k}\right)$ starts dominating over
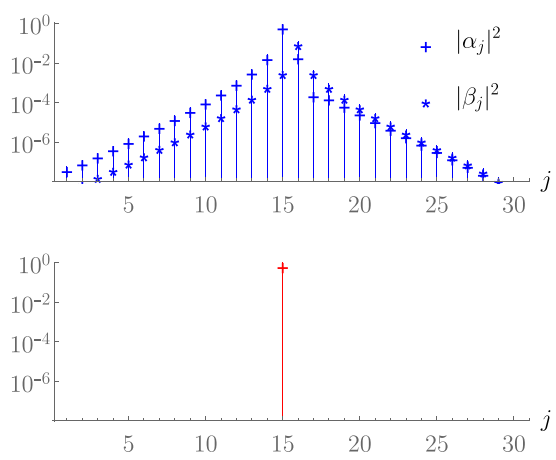

(a)

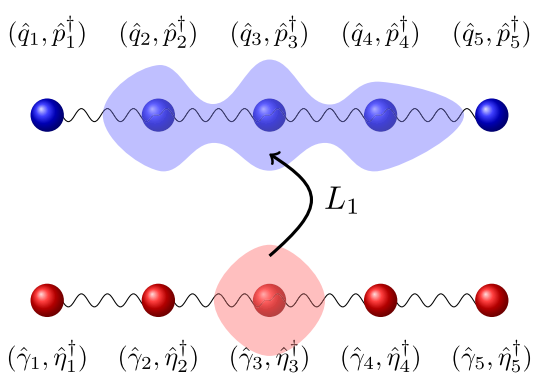

(b)
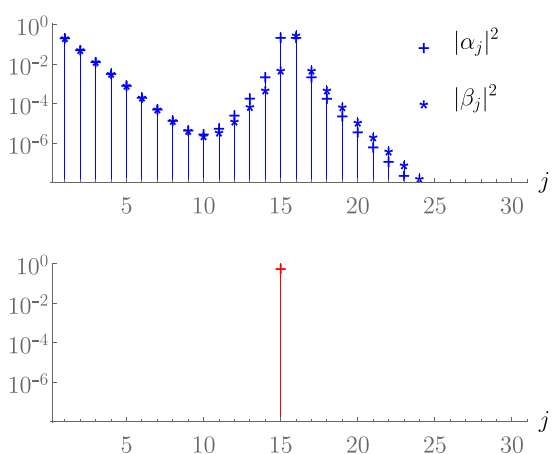

(c)

FIG. 2. Locality of the identification map $L_{1}$ and its dependence on the relative coupling $t / \mu$. As schematically visualized in (b), the plots show, for a system of $N=30$ modes, how $L_{1}$ associates the onsite operator $\hat{c}_{15}$ in the fermionic Kitaev chain in Eq. (53) to the operator $L_{1}\left(\hat{c}_{15}\right)=\sum_{j} \alpha_{j} \hat{b}_{j}+\beta_{j} \hat{b}_{j}^{\dagger}$ on the bosonic Kane-Lubensky chain in Eq. (55). In the trivial phase, as plotted on the left in (a) for $t=0.35 \mu$, the identification map preserves locality to a very high degree, namely, with an exponential decay of the coefficients $\left|\alpha_{j}\right|^{2}$ and $\left|\beta_{j}\right|^{2}$ with the distance $|k-j|$ (here $k \equiv 15$ ). In the topological phase, as plotted on the right in (c) for $t=\mu$, the operator $L_{1}\left(\hat{c}_{k}\right)$ can be nonlocal with a strong contribution from the boundary sites. 
the bulk coming from (bosonic) sites in the neighborhood of the $k$ th site.

Instead of the map $L_{1}$, we may as well employ $L_{2}^{-1}$ to map the fermionic site operators to their bosonic counterparts. The observed behavior is similar; however, for $L_{2}^{-1}\left(\hat{c}_{k}\right)$, in the topological phase, the edge contribution at the left end of the bosonic chain dominates as $k \rightarrow N$, i.e., when the original fermionic operator approaches the right end of the chain.

The converse association of bosonic onsite operators with the corresponding fermionic observables via the identification maps $L_{2}$ or $L_{1}^{-1}$ behaves very similar: In the trivial phase, they are exponentially localized as above, and in the topological phase, they exhibit similar nonlocal features. However, here, both $L_{2}\left(\hat{b}_{k}\right)$ and $L_{1}^{-1}\left(\hat{b}_{k}\right)$ develop a dominant edge contribution when the original bosonic operator $\hat{b}_{k}$ approaches the left end of the chain. For $L_{2}\left(\hat{b}_{k}\right)$, the edge contribution appears on the left edge of the fermionic chain; for $L_{1}^{-1}\left(\hat{b}_{k}\right)$, it appears on the right edge.

This example demonstrates that the identification between the bosonic and the fermionic parts of a SUSY Gaussian state via the identification maps may or may not coincide with an identification based intuitively on some underlying (lattice) geometry of the SUSY Hamiltonians. Whereas we observe agreement in the trivial phase of the SUSY Kitaev chain, in the topological phase, the identification maps behave vastly differently and disengage from notions based on the geometric intuition. The duality relations of the next section will show that, whereas the geometrical appearance of modes can be distorted by the identification maps, their entanglement properties remain intimately related.

\section{ENTANGLEMENT DUALITY}

In this section, we derive how subsystem decompositions $V=A \oplus B$ behave under the supersymmetric identification maps $L_{1}$ and $L_{2}$, which leads to a duality between the bosonic and fermionic (mixed) Gaussian states. We can also use this to relate the associated entanglement entropies.

\section{A. Reduced Gaussian states and entanglement}

Given a classical phase space $V \simeq \mathbb{R}^{2 N}$, a subspace $A \subset$ $V$ defines a physical subsystem if the following condition is satisfied:

(1) Bosonic: The restriction of $\Omega^{a b}$ to the subspace $A$ is nondegenerate, i.e., has nonzero determinant.

(2) Fermionic: The subspace $A$ is even dimensional.

Note that the bosonic condition also implies that $A$ is even dimensional, as any antisymmetric odd-dimensional matrix has a vanishing determinant.

In practice, we choose a basis $\hat{\xi}^{a}=\left(\hat{\xi}_{A}^{a}, \hat{\xi}_{B}^{a}\right)$ that splits $V=$ $A \oplus B$ into a direct sum, where $B$ is the complementary system to $A$ defined as

$$
B=\left\{\begin{array}{ll}
\left\{v^{a} \in V \mid v^{a} \Omega_{a b}^{-1} u^{b}=0 \forall u^{b} \in A\right\} & \text { (bosons) } \\
\left\{v^{a} \in V \mid v^{a} G_{a b}^{-1} u^{b}=0 \forall u^{b} \in A\right\} & \text { (fermions) }
\end{array},\right.
$$

which is called the symplectic complement for bosons and the orthogonal complement for fermions. ${ }^{8}$ We have the two bases $\hat{\xi}_{A}$ and $\hat{\xi}_{B}$ with $N_{A}+N_{B}=N$, such that the resulting matrix representations of $\Omega^{a b}$ and $G^{a b}$ take the forms

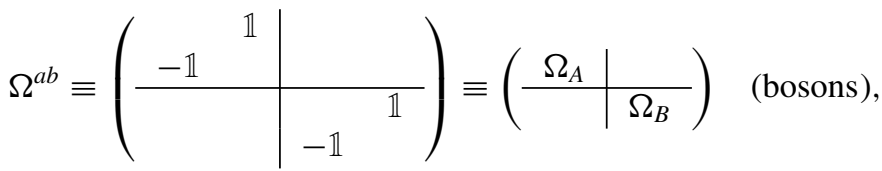

$$
\begin{aligned}
& G^{a b} \equiv\left(\begin{array}{ll|ll}
\mathbb{1} & & & \\
& \mathbb{1} & & \\
\hline & & \mathbb{1} & \\
& & \mathbb{1}
\end{array}\right) \equiv\left(\begin{array}{l|l}
G_{A} & \\
\hline & G_{B}
\end{array}\right) \quad \text { (fermions). }
\end{aligned}
$$

Note that this implies that the restrictions $\Omega_{A}$ and $\Omega_{B}$, or $G_{A}$ and $G_{B}$, respectively, reproduce the standard forms from Eq. (9), i.e.,the subsystems are themselves a bosonic or fermionic system consisting of $N_{A}$ and $N_{B}$ degrees of freedom.

When quantizing the subsystems $A$ and $B$, we can construct Fock spaces $\mathcal{H}_{A}$ and $\mathcal{H}_{B}$ as described in Sec. II A, such that the full Hilbert space is a tensor product $\mathcal{H}=\mathcal{H}_{A} \otimes \mathcal{H}_{B}$. In general, a pure Gaussian state $|J\rangle \in \mathcal{H}$ will itself not be a

\footnotetext{
${ }^{8}$ Here, we used the inverse matrices $\Omega^{-1}$ and $G^{-1}$, which are bilinear forms on the phase space (rather than its dual). In Refs. [63,64,69], they are denoted by $\Omega_{a b}^{-1} \equiv \omega_{a b}$ and $G_{a b}^{-1} \equiv g_{a b}$.
}

tensor product state with respect to this decomposition, which means that the subsystems are entangled.

It is well-known that the bipartite entanglement encoded in a general pure state $|\psi\rangle$ can be characterized by the spectrum of the mixed state $\rho_{A}=\operatorname{Tr}_{B}|\psi\rangle\langle\psi|$ that results from tracing over $\mathcal{H}_{B}$. If $|\psi\rangle$ is a pure Gaussian state $|J\rangle$, the reduced state $\rho_{A}$ is a mixed Gaussian state. It can be expressed in terms of the linear complex structure $J$ as [63]

$$
\rho_{A}=e^{-\hat{Q}} \quad \text { with } \quad \hat{Q}= \begin{cases}q_{r s} \hat{\xi}^{r} \hat{\xi}^{s}+c_{0} & \text { (bosons) } \\ \mathrm{i} q_{r s} \hat{\xi}^{r} \hat{\xi}^{s}+c_{0} & \text { (fermions) }\end{cases}
$$


with $q_{r s}$ is a $2 N_{A}$-by- $2 N_{A}$ matrix $^{9}$ given by [63]

$$
\begin{aligned}
q_{r s} & = \begin{cases}-\mathrm{i}\left(\Omega_{A}^{-1}\right)_{r l} \operatorname{arccoth}\left(\mathrm{i}_{A}\right)_{s}^{l} & \text { (bosons) } \\
+\mathrm{i}\left(G_{A}^{-1}\right)_{r l} \operatorname{arctanh}\left(\mathrm{i} J_{A}\right)_{s}^{l} & \text { (fermions) }\end{cases} \\
& =\left\{\begin{array}{ll}
+\left(\Omega_{A}^{-1}\right)_{r l} \operatorname{arccot}\left(J_{A}\right)_{s}^{l} & \text { (bosons) } \\
-\left(G_{A}^{-1}\right)_{r l} \operatorname{arctanh}\left(J_{A}\right)_{s}^{l} & \text { (fermions) }
\end{array} .\right.
\end{aligned}
$$

Here, $J_{A}$ is the restriction of $J$ to the $2 N_{A}$-by-2 $2 N_{A}$ subblock, representing the action of $J$ onto the subspace $A \subset V$, and similarly, $\Omega_{A}^{-1}$ and $G_{A}^{-1}$ denote the restrictions of $\Omega^{-1}$ and $G^{-1}$. The coefficient $c_{0}$ is given by

$$
c_{0}=\left\{\begin{array}{ll}
\frac{1}{4} \log \operatorname{det}\left(\frac{\mathbb{1}+J_{A}^{2}}{4}\right) & \text { (bosons) } \\
-\frac{1}{4} \log \operatorname{det}\left(\frac{\mathbb{1}+J_{A}^{2}}{4}\right) & \text { (fermions) }
\end{array} .\right.
$$

It can be shown $[62,63]$ that the eigenvalues of $J_{A}$ are purely imaginary and appear in $N_{A}$ conjugate pairs $\pm \mathrm{i} \lambda_{i}$, where $\lambda_{i} \in$ $[1, \infty)$ for bosons, and $\lambda_{i} \in[0,1]$ for fermions.

These relations have the consequence that, for Gaussian states, the rather complicated spectrum of $\rho_{A}$ simplifies so that it can be efficiently calculated from the much simpler spectrum of $J_{A}$ given by $\pm \mathrm{i} \lambda_{i}$. Specifically, the eigenvalues of $\rho_{A}$ are

$$
\mu\left(n_{1}, \ldots, n_{N}\right)=\left\{\begin{array}{ll}
{\left[\prod_{i=1}^{N_{A}} \frac{\left(\tanh r_{i}\right)_{i}}{\cosh r_{i}}\right]^{2}} & \text { (bosons) } \\
{\left[\prod_{i=1}^{N_{A}} \frac{\left(\tan r_{i}\right)^{n_{i}}}{\sec r_{i}}\right]^{2}} & \text { (fermions) }
\end{array},\right.
$$

where $r_{i}=\frac{1}{2} \cosh ^{-1}\left(\lambda_{i}\right), \quad n_{i} \in \mathbb{N}$ for bosons, and $r_{i}=$ $\frac{1}{2} \cos ^{-1}\left(\lambda_{i}\right), n_{i}=0,1$ for fermions.

The entanglement entropy $S_{A}(|\psi\rangle)=S_{B}(|\psi\rangle)$ is computed as the von Neumann entropy $S\left(\rho_{A}\right)$ of the reduced state $\rho_{A}$, namely,

$$
S_{A}(|\psi\rangle)=S\left(\rho_{A}\right)=-\operatorname{Tr} \rho_{A} \log \rho_{A} .
$$

Calculating this quantity in practice is notoriously hard, as it requires computing the spectrum of $\rho_{A}$ that demands vast computational resources for large systems and appropriate approximations or truncation for infinite dimensional Hilbert spaces (in the case of bosons). However, if the state $|\psi\rangle$ happens to be a Gaussian state $|J\rangle$, we can exploit the relation between the spectra of $\rho_{A}$ and $J_{A}$ to find analytical formulas in terms of the restriction $J_{A}$ to the subsystem $A$. These restrictions correspond exactly to the symplectic or orthogonal decomposition $V=A \oplus B$ introduced at the beginning of this section. The formulas for the von Neumann entropies are given by $[55,56]$

$$
S\left(\rho_{A}\right)=\left\{\begin{array}{ll}
\sum_{i=1}^{N_{A}} s_{\mathrm{b}}\left(\lambda_{i}\right) & \text { (bosons) } \\
\sum_{i=1}^{N_{A}} s_{\mathrm{f}}\left(\lambda_{i}\right) & \text { (fermions) }
\end{array},\right.
$$

with $s_{\mathrm{b}}(x)=\left(\frac{x+1}{2}\right) \log \left(\frac{x+1}{2}\right)-\left(\frac{x-1}{2}\right) \log \left(\frac{x-1}{2}\right)$ for bosons, and $s_{\mathrm{f}}(x)=-\frac{1+x}{2} \log \left(\frac{1+x}{2}\right)-\frac{1-x}{2} \log \left(\frac{1-x}{2}\right)$ for fermions, which

\footnotetext{
${ }^{9}$ Not to confuse with the quadrature operator $\hat{q}_{i}$, which carries at most one index.
}

can be unified by the single trace formula $[63,70]$

$$
S\left(\rho_{A}\right)=\frac{1}{2}\left|\operatorname{Tr}\left[\left(\frac{1+\mathrm{i} J_{A}}{2}\right) \log \left(\frac{1+\mathrm{i} J_{A}}{2}\right)^{2}\right]\right| .
$$

The formula in Eq. (65) can also be used to compute the Renyi entropy of order $n$ if we replace $s_{\mathrm{b}}$ and $s_{\mathrm{f}}$ by the respective Renyi entropy functions [63]:

$$
\begin{aligned}
r_{\mathrm{b}}^{(k)}(\lambda) & =\frac{1}{k-1} \log \left[\left(\frac{\lambda+1}{2}\right)^{k}-\left(\frac{\lambda-1}{2}\right)^{k}\right], \\
r_{\mathrm{f}}^{(k)}(\lambda) & =-\frac{1}{k-1} \log \left[\left(\frac{1+\lambda}{2}\right)^{k}+\left(\frac{1-\lambda}{2}\right)^{k}\right] .
\end{aligned}
$$

It follows from the above that a subsystem $A$ (bosonic or fermionic) of a system in a pure Gaussian state is not entangled with the rest of the system, i.e.,it is in a product state with the rest of the system, if and only if $\lambda_{i}=1$ for all eigenvalues of $J_{A}$. In that case, we have $J_{A}^{2}=-\mathbb{1}_{A}$, and the subsystem is in a pure Gaussian state on its own. This is equivalent to $J(A)=A$, i.e.,the full (unrestricted) linear complex structure mapping $A$ onto itself.

\section{B. Supersymmetric ground states and identification maps}

Above in Eq. (42), we saw that $L_{1}$ and $L_{2}$ together encode the linear complex structures of both the bosonic and the fermionic part of the ground state in Eq. (32) of $\hat{H}$. In the following, we will use $L_{1}$ and $L_{2}$ to identify subsystems of fermionic modes with subsystems of bosonic modes and vice versa.

The maps $L_{1}$ and $L_{2}$ are the canonical choices for the identification maps because they preserve the Kähler structures of the fermionic ground state $\left|J_{\mathrm{f}}\right\rangle$ and the bosonic ground state $\left|J_{\mathrm{b}}\right\rangle$. That is, if we consider the fermionic 2-point function

$$
C_{\mathrm{f}, 2}^{\alpha \beta}=\left\langle J_{\mathrm{f}}\left|\hat{\xi}^{\alpha} \hat{\xi}^{\beta}\right| J_{\mathrm{f}}\right\rangle=\frac{1}{2}\left(G_{\mathrm{f}}^{\alpha \beta}+\mathrm{i} \Omega_{\mathrm{f}}^{\alpha \beta}\right),
$$

and the bosonic

$$
C_{\mathrm{b}, 2}^{a b}=\left\langle J_{\mathrm{b}}\left|\hat{\xi}^{a} \hat{\xi}^{b}\right| J_{\mathrm{b}}\right\rangle=\frac{1}{2}\left(G_{\mathrm{b}}^{a b}+\mathrm{i} \Omega_{\mathrm{b}}^{a b}\right),
$$

then one can show that we have

$$
G_{\mathrm{b}}^{a b}=\left(L_{2}\right)^{a}{ }_{\alpha} G_{\mathrm{f}}^{\alpha \beta}\left(L_{2}^{\top}\right)_{\beta}{ }^{b}=\left(L_{1}^{-1}\right)^{a}{ }_{\alpha} G_{\mathrm{f}}^{\alpha \beta}\left(L_{1}^{\top-1}\right)_{\beta}{ }^{b},
$$

as well as (dropping the indices for a better readability)

$$
\Omega_{\mathrm{f}}=L_{1} \Omega_{\mathrm{b}} L_{1}^{\top}=L_{2}^{-1} \Omega_{\mathrm{b}} L_{2}^{-1 \top} .
$$

Thus, the identification maps $L_{1}$ and $L_{2}$ preserve both the symmetric and the antisymmetric forms of the Kähler structure and exactly map the bosonic and fermionic 2-point functions of the ground state onto each other. Interestingly, we see that it makes no difference whether we use $L_{1}$ and $\left(L_{1}\right)^{-1}$ or $L_{2}$ and $\left(L_{2}\right)^{-1}$ for this purpose. The reason is that both maps are closely related. In fact, since $J_{\mathrm{f}}^{2}=-\mathbb{1}$ and $J_{\mathrm{b}}^{2}=-\mathbb{1}$, it follows that

$$
\begin{aligned}
& \left(L_{1}\right)^{-1}=-L_{2} J_{\mathrm{f}}=-J_{\mathrm{b}} L_{2}, \\
& \left(L_{2}\right)^{-1}=-J_{\mathrm{f}} L_{1}=-L_{1} J_{\mathrm{b}} .
\end{aligned}
$$




\section{Dual supersymmetric subsystems}

Since the identification maps $L_{1}$ and $L_{2}$ preserve the Kähler structures, subsystems in one part (bosonic/fermionic) of a supersymmetric Gaussian state can be identified with subsystems in the other part (fermionic/bosonic).

If $A \subset V_{\mathrm{b}}$ corresponds to a bosonic subsystem, then both $L_{1}(A)$ and $L_{2}^{-1}(A)$ are even-dimensional subspaces of the fermionic phase space $V_{\mathrm{f}}$; hence, they correspond to a fermionic subsystem, as defined in Sec. III A. If, on the other hand, $A \subset V_{\mathrm{f}}$ corresponds to a fermionic subsystem, then $L_{2}(A)$ and $L_{1}^{-1}(A)$ only correspond to a bosonic subsystem, if the restriction of $\Omega_{\mathrm{f}}$ to $A$ is nondegenerate. Following Eq. (72), this condition ensures that $\Omega_{\mathrm{b}}$ is nondegenerate as required for $L_{2}(A)$ and $L_{1}^{-1}(A)$ to yield a bosonic subsystem.

How does the subsystem which $A$ is mapped to depend on whether we use the identification map $L_{1}$ (and its inverse) or the map $L_{2}$ ? If the subsystem $A$ is in a pure state, there is no difference; both identification maps identify $A$ with the same subsystem. For example, if $A \subset V_{\mathrm{b}}$ is a bosonic subsystem which is in a pure state, then we have $J_{\mathrm{b}}(A)=A$; thus,

$$
L_{1}(A)=L_{1}\left[J_{\mathrm{b}}(A)\right]=L_{2}^{-1}(A) .
$$

However, for an entangled subsystem, we have $J(A) \neq A$ and are led to the following commutative diagram:

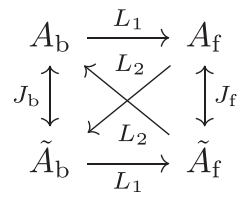

Here, we have chosen $A_{\mathrm{b}} \subset V_{\mathrm{b}}$ as a bosonic subsystem, defined $A_{\mathrm{f}}=L_{1}\left(A_{\mathrm{b}}\right)$ and denoted $\tilde{A}_{\mathrm{b}}=J_{\mathrm{b}}\left(A_{\mathrm{b}}\right)$ and $\tilde{A}_{\mathrm{f}}=J_{\mathrm{f}}\left(A_{\mathrm{f}}\right)$.

Whereas $A_{\mathrm{b}}$ and $\tilde{A}_{\mathrm{b}}\left(A_{\mathrm{f}}\right.$ and $\left.\tilde{A}_{\mathrm{f}}\right)$ define different bosonic (fermionic) subsystems, they are intimately related: $A_{\mathrm{b}} \cup \tilde{A}_{\mathrm{b}}$ $\left(A_{\mathrm{f}} \cup \tilde{A}_{\mathrm{f}}\right)$ is the smallest subsystem containing $A_{\mathrm{b}}\left(A_{\mathrm{f}}\right)$ which is in a pure partial state, i.e.,shares no entanglement with the rest of the system.

Furthermore, $A_{\mathrm{b}}\left(A_{\mathrm{f}}\right)$ shares the same amount of entanglement with the rest of the system as does $\tilde{A}_{\mathrm{b}}\left(\tilde{A}_{\mathrm{f}}\right)$. This follows from the fact that the restricted linear complex structures $J_{A_{\mathrm{b}}}^{\mathrm{b}}$ and $J_{\tilde{A}_{\mathrm{b}}}^{\mathrm{b}}\left(J_{A_{\mathrm{f}}}^{\mathrm{f}}\right.$ and $\left.J_{\tilde{A}_{\mathrm{f}}}^{\mathrm{f}}\right)$ have the same spectrum. To see this, consider the decomposition of the phase space into the direct sum $V_{\mathrm{b}}=A_{\mathrm{b}} \oplus B_{\mathrm{b}}$ according to Eq. (58). We define by $P_{A_{\mathrm{b}}}$ the projector onto $A$ with respect to this decomposition:

$$
P_{A_{\mathrm{b}}}\left(A_{\mathrm{b}}\right)=A_{\mathrm{b}}, \quad P_{A_{\mathrm{b}}}\left(B_{\mathrm{b}}\right)=0 .
$$

The restriction of $J_{\mathrm{b}}$ to $A_{\mathrm{b}}$ is then $J_{A_{\mathrm{b}}}^{\mathrm{b}}=P_{A_{\mathrm{b}}} J_{\mathrm{b}} P_{A_{\mathrm{b}}}$. Analogously, considering the decomposition $V_{\mathrm{b}}=\tilde{A}_{\mathrm{b}} \oplus \tilde{B}_{\mathrm{b}}$, we find that the projector onto $\tilde{A}_{\mathrm{b}}$ is $P_{\tilde{A}_{\mathrm{b}}}=-J_{\mathrm{b}} P_{A_{\mathrm{b}}} J_{\mathrm{b}}$, and

$$
J_{\tilde{A}_{\mathrm{b}}}^{\mathrm{b}}=P_{\tilde{A}_{\mathrm{b}}} J_{\mathrm{b}} P_{\tilde{A}_{\mathrm{b}}}=-J_{\mathrm{b}} J_{A_{\mathrm{b}}}^{\mathrm{b}} J_{\mathrm{b}}
$$

Since $J_{\mathrm{b}}^{-1}=-J_{\mathrm{b}}, J_{\tilde{A}_{\mathrm{b}}}^{\mathrm{b}}$ and $J_{\tilde{A}_{\mathrm{b}}}^{\mathrm{b}}$ are represented by similar matrices and, hence, have the same spectrum. In fact, if $v \in A_{\mathrm{b}}$ is an eigenvector of $J_{A_{\mathrm{b}}}^{\mathrm{b}}$ with $J_{A_{\mathrm{b}}}^{\mathrm{b}} v= \pm i \lambda v$, then $J_{\mathrm{b}} v$ is an eigenvector of $J_{\tilde{A}_{\mathrm{b}}}^{\mathrm{b}}$ with the same eigenvalue.

\section{Duality for Gaussian states and their entanglement}

In the previous section, we analyzed the structure of subsystems in supersymmetric Gaussian states. We discussed how the identification maps $L_{1}$ and $L_{2}$ relate bosonic subsystems to fermionic subsystems and vice versa. We can now use this background structure to derive the following duality between bosonic and fermionic Gaussian states.

The setting is as follows. We consider a classical phase space $V \simeq \mathbb{R}^{2 N}$ with Kähler compatible structures $(G, \Omega, J)$ and a choice of a subspace $A \subset V$ with $\operatorname{dim} A=2 N_{A}$. We can associate two distinct quantum theories, namely, a bosonic Hilbert space $\mathcal{H}_{\mathrm{b}}$ with Gaussian state $|J\rangle_{\mathrm{b}}$ and a fermionic Hilbert space $\mathcal{H}_{\mathrm{f}}$ with Gaussian state $|J\rangle_{\mathrm{f}}$. In both quantum theories, we can construct a reduced density operator $\rho_{A}$ whose spectrum is determined by the restricted complex structure.

Crucially, however, the restriction of $J$ to $A$ is different depending on whether we consider a bosonic system and use a symplectic decomposition of the phase space or consider a fermionic system and use an orthogonal decomposition, according to Eq. (58). This is due to the fact that the $2 N_{A}$-by- $2 N_{A}$ subblock of the matrix $J$ associated to the subspace $A$ depends also on the basis elements that are not contained in $A$. We choose two different bases for the bosonic and fermionic case $\hat{\xi}_{\mathrm{b}}=\left(\hat{\xi}_{\mathrm{b}}^{A}, \hat{\xi}_{\mathrm{b}}^{B}\right)$ and $\hat{\xi}_{\mathrm{f}}=\left(\hat{\xi}_{\mathrm{f}}^{A}, \hat{\xi}_{\mathrm{f}}^{B}\right)$, such that

$$
\begin{gathered}
\operatorname{span}\left(\hat{\xi}_{\mathrm{b}}^{A}\right)=A=\operatorname{span}\left(\hat{\xi}_{\mathrm{f}}^{A}\right), \\
\operatorname{span}\left(\hat{\xi}_{\mathrm{b}}^{B}\right)=B_{\mathrm{b}} \neq B_{\mathrm{f}}=\operatorname{span}\left(\hat{\xi}_{\mathrm{f}}^{B}\right),
\end{gathered}
$$

where $B_{\mathrm{b}}$ and $B_{\mathrm{f}}$ are the respective bosonic and fermionic complements defined in Eq. (58). Consequently, the restrictions of $J$ to the subspace $A$ can be different on the bosonic and the fermionic side, which therefore are denoted by $J_{A}^{\mathrm{b}}$ and $J_{A}^{\mathrm{f}}$, respectively. Equipped with this, we can now prove the following proposition.

Proposition 1 (Entanglement duality). We consider a supersymmetric system with phase space $V \simeq V_{\mathrm{b}} \simeq V_{\mathrm{f}}$ equipped with Kähler structures $(G, \Omega, J)$, which simultaneously describe a bosonic and a fermionic Gaussian state, namely, $|J\rangle_{\mathrm{b}} \in \mathcal{H}_{\mathrm{b}}$ and $|J\rangle_{\mathrm{f}} \in \mathcal{H}_{\mathrm{f}}$. We now choose a subsystem $A \subset$ $V$. This leads to two inequivalent decompositions of $V$, namely, $V=A \oplus B_{\mathrm{b}}$ and $V=A \oplus B_{\mathrm{f}}$, where the complementary subsystems $B_{\mathrm{b}}$ and $B_{\mathrm{f}}$ are defined in Eq. (58). The associated reduced states $\rho_{A}^{\mathrm{b}}$ and $\rho_{A}^{\mathrm{f}}$ are both Gaussian and fully described by the restricted complex structure $J_{A}^{\mathrm{b}}$ and $J_{A}^{\mathrm{f}}$, respectively, which satisfy the following relation:

$$
J_{A}^{\mathrm{f}}=-\left(J_{A}^{\mathrm{b}}\right)^{-1} \text {. }
$$

This implies that the eigenvalues $\pm \mathrm{i} \lambda_{i}^{\mathrm{b}}$ of $J_{A}^{\mathrm{b}}$ are related to the eigenvalues $\pm \mathrm{i} \lambda_{i}^{\mathrm{f}}$ of $J_{A}^{\mathrm{f}}$ via $\lambda_{i}^{\mathrm{b}}=1 / \lambda_{i}^{\mathrm{f}}$.

Proof. The decompositions $V=A \oplus B_{\mathrm{b}}$ and $V=A \oplus B_{\mathrm{f}}$ define projectors, such that $P_{\mathrm{b}}: V \rightarrow A, P_{\mathrm{f}}: V \rightarrow A, \bar{P}_{\mathrm{b}}$ : $V \rightarrow B_{\mathrm{b}}$, and $\bar{P}_{\mathrm{f}}: V \rightarrow A_{\mathrm{f}}$, such that $\mathbb{1}=P_{\mathrm{b}}+\bar{P}_{\mathrm{b}}=P_{\mathrm{f}}+\bar{P}_{\mathrm{f}}$. The restricted complex structures are then defined as

$$
\begin{gathered}
J_{A}^{\mathrm{b}}=\left.P_{\mathrm{b}} J\right|_{A}: A \rightarrow A, \\
J_{A}^{\mathrm{f}}=\left.P_{\mathrm{f}} J\right|_{A}: A \rightarrow A .
\end{gathered}
$$


We need to show $J_{A}^{\mathrm{f}}=-\left(J_{A}^{\mathrm{b}}\right)^{-1}$, which is equivalent to $J_{A}^{\mathrm{f}} J_{A}^{\mathrm{b}}=$ $-\mathbb{1}_{A}$. To show the latter, we take a vector $a \in A$ and calculate

$$
\begin{aligned}
-a & =-P_{\mathrm{f}} a=P_{\mathrm{f}} J^{2} a=P_{\mathrm{f}} J\left(P_{\mathrm{b}}+\bar{P}_{\mathrm{b}}\right) J a \\
& =P_{\mathrm{f}} J J_{A}^{\mathrm{b}} a+P_{\mathrm{f}} J \bar{P}_{\mathrm{b}} J a=J_{A}^{\mathrm{f}} J_{A}^{\mathrm{b}} a+P_{\mathrm{f}} J \bar{P}_{\mathrm{b}} J a .
\end{aligned}
$$

The second term in Eq. (83) vanishes since, for an arbitrary vector $v \in V$, the inner product

$$
\begin{aligned}
G^{-1}\left(v, P_{\mathrm{f}} J \bar{P}_{\mathrm{b}} a\right) & =G^{-1}\left(P_{\mathrm{f}} v, J \bar{P}_{\mathrm{b}} J a\right) \\
& =-\Omega^{-1}(\underbrace{P_{\mathrm{f}} v}_{\in A}, \underbrace{\bar{P}_{\mathrm{b}} J a}_{B_{\mathrm{b}}})=0,
\end{aligned}
$$

where we have used the relationship $G^{-1}(\cdot, J \cdot)=-\Omega^{-1}(\cdot, \cdot)$ following from Eq. (11). In matrix notation, we would write $G^{-1}(v, w)=\left(G^{-1}\right)_{a b} v^{a} w^{b}$ and so on. That the inner product $\Omega^{-1}(\cdot, \cdot)$ in Eq. (84) vanishes follows from the definition of $B_{\mathrm{b}}$ in Eq. (58), and therefore, proves the identity in Eq. (80).

At first glance, this result is a simple statement about restricting a complex structure $J: V \rightarrow V$ to a subspace $A \subset V$ in two inequivalent ways. However, its application to bosonic and fermionic Gaussian states implies a rather complicated relationship of the spectra $\rho_{A}^{\mathrm{b}}$ and $\rho_{A}^{\mathrm{f}}$ via Eqs. (63) and (80), which can be made precise in the following corollary relating the restricted complex structures of the dual subsystems.

Corollary 1. Given a supersymmetric system with supercharge operator $\hat{\mathcal{Q}}$, we have a supersymmetric ground state $\left|J_{\mathrm{b}}\right\rangle \otimes\left|J_{\mathrm{f}}\right\rangle$ of $\hat{H}=\hat{\mathcal{Q}}^{2}$ and identification maps $L_{1}: V_{\mathrm{b}} \rightarrow V_{\mathrm{f}}$, $L_{2}: V_{\mathrm{f}} \rightarrow V_{\mathrm{b}}$, and their inverses $L_{1}^{-1}$ and $L_{2}^{-1}$, as above. Then Proposition 1 implies the following.

Let $S \subset V_{\mathrm{b}}$ be a bosonic subsystem and $L(S) \subset V_{\mathrm{f}}$, with $L=L_{1}$ or $L=L_{2}^{-1}$, be a dual fermionic subsystem. Then the restricted linear structures $J_{S}^{\mathrm{b}}: S \rightarrow S$ and $J_{L(S)}^{\mathrm{f}}: L(S) \rightarrow$ $L(S)$ of these two subsystems are such that

$$
\left(J_{S}^{\mathrm{b}}\right)^{-1}=-L^{-1} J_{L(S)}^{\mathrm{f}} L, \quad\left(J_{L(S)}^{\mathrm{f}}\right)^{-1}=-L J_{S}^{\mathrm{b}} L^{-1} .
$$

Let $R \subset V_{\mathrm{f}}$ be a fermionic subsystem and $L(R) \subset V_{\mathrm{b}}$, with $L=L_{2}$ or $L=L_{1}^{-1}$, be a dual bosonic subsystem. Then the restricted linear structures $J_{R}^{\mathrm{f}}: R \rightarrow R$ and $J_{L(R)}^{\mathrm{b}}: L(R) \rightarrow L(R)$ of these two subsystems are such that

$$
\left(J_{R}^{\mathrm{f}}\right)^{-1}=-L^{-1} J_{L(R)}^{\mathrm{b}} L, \quad\left(J_{L(R)}^{\mathrm{b}}\right)^{-1}=-L J_{R}^{\mathrm{f}} L^{-1} .
$$

Thus, the eigenvalues of the dual restricted complex structures are inverses of each other as implied by Eq. (80), and their entanglement spectra are accordingly related by Eq. (63).

While our result applies to any identification where a bosonic and a fermionic phase space are related, supersymmetric systems with the identification maps $L_{1}$ and $L_{2}$, as discussed in Sec. IIC, are the prime examples where such an identification is naturally chosen.

The entanglement duality implies an intimate relation of the entanglement entropy of a subsystem with that of its dual subsystem because both the von Neumann entropy in Eq. (65), as well as the Renyi entropies in Eq. (67) are functions of the spectrum of the restricted complex linear structure. For the simplest possible case, where the subsystems each consist of a single mode only, Fig. 3 shows the relation between the von Neumann entropy of the fermionic and the bosonic modes. Here, the restricted complex structures have one pair

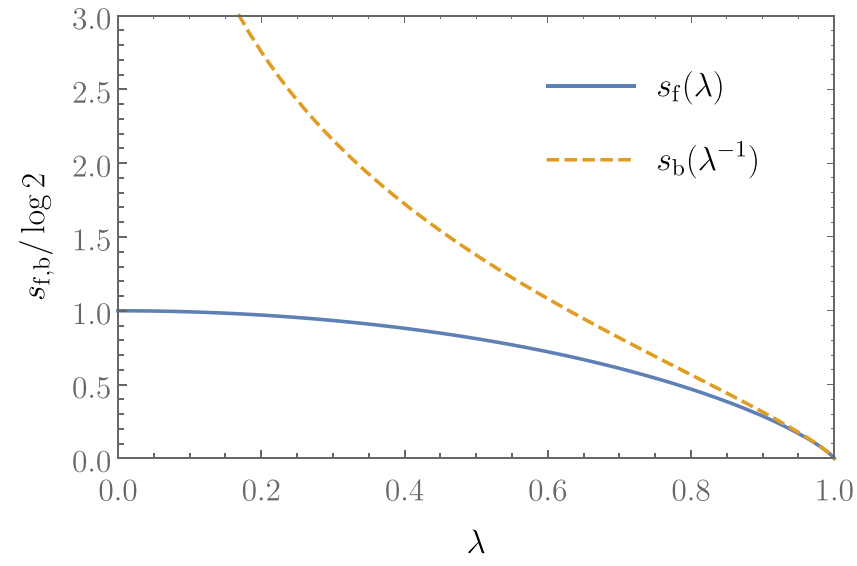

FIG. 3. Entanglement entropies for dual subsystems each consisting of a single mode. The solid line shows the entanglement entropy of a single fermionic mode for which the restricted complex structure has eigenvalues $\pm \mathrm{i} \lambda$. Due to the entanglement duality in Eq. (80), the restricted complex structure of the dual bosonic mode has eigenvalues $\pm \mathrm{i} \lambda^{-1}$, and the dashed line plots the resulting entanglement entropy.

of imaginary eigenvalues, $\pm \mathrm{i} \lambda$ for the fermionic and $\pm \mathrm{i} \lambda^{-1}$ for the bosonic system, which with the formula for the von Neumann entropies in Eq. (65) yields the relation plotted in Fig. 3.

Evidently, the bosonic and the fermionic entanglement become asymptotically equal when the corresponding modes approach a pure partial state, and consequently, the entanglement approaches zero $(\lambda \rightarrow 1)$. In the opposite direction, however, the entanglement in the bosonic mode grows without a bound as $\lambda \rightarrow 0$, whereas the entanglement in the dual fermionic mode tends to saturate at the maximal value of $\log 2$.

This relation between the SUSY partner single modes readily extends to multiple modes because, as is evident from Eq. (65), the total entanglement entropy of a subsystem is given by the sum of the entanglement entropies over the individual normal modes of that subsystem. This is related to the fact that a mixed Gaussian state always can be expressed as the product state of its normal modes, which are given by the eigenmodes of the restricted linear complex structure [62]. As a consequence of the entanglement duality, the identification maps identify normal modes with reciprocal eigenvalues of the restricted complex linear structures.

At this stage, it is an important question whether the entanglement duality is merely an interesting observation or to what extent it matters for physical systems. In the following two sections, we therefore investigate the entanglement duality in two concrete applications. First, we consider the toy model of a supersymmetric system with two bosonic and two fermionic modes in Sec. IIIE before we then move onto the recently proposed SUSY Kitaev honeycomb model in Sec. III F.

Before proceeding, we note that mixed states that arise as a thermal state of a supersymmetric Hamiltonian also come under the ambit of our duality. In detail, we ask if a relation, like what applies to the mixed states arising from a reduction of a pure state to a subsystem $A$, holds for a thermal state of a supersymmetric Hamiltonian $\hat{H}=\hat{\mathcal{Q}}^{2}=\hat{H}_{\mathrm{b}}+\hat{H}_{\mathrm{f}}$, 
i.e., $\rho=\frac{1}{Z} e^{\beta \hat{H}}=\frac{1}{Z} e^{\beta \hat{H}_{\mathrm{b}}} \otimes e^{\beta \hat{H}_{\mathrm{f}}}$. The following proposition answers this question in the affirmative.

Proposition 2 (Thermal state duality). We consider a supersymmetric system with phase space $V \simeq V_{\mathrm{b}} \simeq V_{\mathrm{f}}$ equipped with Kähler structures $(G, \Omega, J)$, for which we have a Hamiltonian $\hat{H}=\hat{\mathcal{Q}}^{2}=\hat{H}_{\mathrm{b}}+\hat{H}_{\mathrm{f}}$. The thermal state $\rho=\frac{1}{Z} e^{\beta \hat{H}}$ at inverse temperature $\beta$ is a tensor product of two Gaussian states $\rho=\rho_{\mathrm{b}} \otimes \rho_{\mathrm{f}}$ with associated restricted complex structures $J_{\mathrm{b}}$ and $J_{\mathrm{f}}$ related by

$$
J_{\mathrm{b}}=-\left(J_{\mathrm{f}}\right)^{-1}
$$

which exactly resembles the entanglement duality, but now applies to the whole system. This implies that the eigenvalues $\pm \mathrm{i} \lambda_{i}^{\mathrm{b}}$ of $J_{\mathrm{b}}$ are related to the eigenvalues $\pm \mathrm{i} \lambda_{i}^{\mathrm{f}}$ of $J_{\mathrm{f}}$ via $\lambda_{i}^{\mathrm{b}}=$ $1 / \lambda_{i}^{\mathrm{f}}$.

Proof. Our identification of the phase spaces $V \simeq V_{\mathrm{b}} \simeq$ $V_{\mathrm{f}}$ gives rise to a single Lie algebra generator $K: V \rightarrow V$ for the Hamiltonian $\beta \hat{H}$ from Eq. (25). The spectrum of $K$ agrees with that of the bosonic generator $K_{\mathrm{b}}: V_{\mathrm{b}} \rightarrow V_{\mathrm{b}}$ as well as the fermionic generator $K_{\mathrm{f}}: V_{\mathrm{f}} \rightarrow V_{\mathrm{f}}$ defined as $\left(K_{\mathrm{b}}\right)^{a}{ }_{b}=$ $\beta \Omega^{a c} h_{c b}^{\mathrm{b}}$ and $\left(K_{\mathrm{f}}\right)^{\alpha}{ }_{\delta}=\beta G^{\alpha \gamma} q_{\gamma \delta}^{\mathrm{f}}$, respectively. We can compare with Eq. (60) to identify that $\rho=e^{-\beta \hat{H}} / Z$ gives rise to $q_{a b}^{\mathrm{b}}=\frac{\beta}{2} h_{a b}^{\mathrm{b}}=\frac{\beta}{2} \Omega_{a c}^{-1}\left(K_{\mathrm{b}}\right)^{c}{ }_{b}$ and $q_{\alpha \gamma}^{\mathrm{f}}=\frac{\beta}{2} h_{\alpha \gamma}^{\mathrm{f}}=\frac{\beta}{2} G_{\alpha \delta}^{-1}\left(K_{\mathrm{f}}\right)^{\delta}{ }_{\gamma}$. We can invert Eq. (61) to find

$$
\begin{gathered}
J_{\mathrm{b}}=-\cot \Omega q^{\mathrm{b}}=-\cot \left(\frac{K_{\mathrm{b}}}{2}\right) \equiv-\cot \left(\frac{K}{2}\right), \\
J_{\mathrm{f}}=\tan G q^{\mathrm{f}}=\tan \left(\frac{K_{\mathrm{f}}}{2}\right) \equiv \tan \left(\frac{K}{2}\right),
\end{gathered}
$$

from which Eq. (87) readily follows.

\section{E. Application: two-mode system}

In this section, we study some consequences of the entanglement duality in a basic two-mode example where the
SUSY Hamiltonian is given by a fermionic and a bosonic two-mode squeezing Hamiltonian. While this is a minimal example, it explains certain basic relations which are important for our analysis of a lattice Hamiltonian in the next subsection. It highlights that almost maximally entangled fermionic modes are mapped to almost degenerate bosonic modes. This relation is central to the entanglement scaling in $2 \mathrm{D}$ systems discussed in Sec. III F.

Consider the following supercharge operator $\hat{\mathcal{Q}}$, which is parametrized by real numbers $r_{\mathrm{b}} \geqslant 0$ and $0 \leqslant r_{\mathrm{f}}<\pi / 4$, corresponding to squeezing parameters:

$$
\begin{aligned}
\hat{\mathcal{Q}}= & {\left[\cosh \left(r_{\mathrm{b}}\right) \cos \left(r_{\mathrm{f}}\right)-\sinh \left(r_{\mathrm{b}}\right) \sin \left(r_{\mathrm{f}}\right)\right]\left(\hat{\gamma}_{1} \hat{q}_{1}+\hat{\eta}_{1} \hat{p}_{1}\right) } \\
& +\left[\cosh \left(r_{\mathrm{b}}\right) \cos \left(r_{\mathrm{f}}\right)+\sinh \left(r_{\mathrm{b}}\right) \sin \left(r_{\mathrm{f}}\right)\right]\left(\hat{\gamma}_{2} \hat{q}_{2}+\hat{\eta}_{2} \hat{p}_{2}\right) \\
& +\left[\cosh \left(r_{\mathrm{b}}\right) \sin \left(r_{\mathrm{f}}\right)-\sinh \left(r_{\mathrm{b}}\right) \cos \left(r_{\mathrm{f}}\right)\right]\left(\hat{\gamma}_{1} \hat{q}_{2}-\hat{\eta}_{1} \hat{p}_{2}\right) \\
& +\left[\cosh \left(r_{\mathrm{b}}\right) \sin \left(r_{\mathrm{f}}\right)+\sinh \left(r_{\mathrm{b}}\right) \cos \left(r_{\mathrm{f}}\right)\right]\left(-\hat{\gamma}_{2} \hat{q}_{1}+\eta_{2} \hat{p}_{1}\right) .
\end{aligned}
$$

It generates a SUSY Hamiltonian $\hat{H}=\hat{H}_{\mathrm{b}}+\hat{H}_{\mathrm{f}}$ which consists of the two-mode Hamiltonians:

$$
\hat{H}_{\mathrm{b}}=\frac{\cosh \left(2 r_{\mathrm{b}}\right)}{2} \sum_{i=1,2}\left(\hat{q}_{i}^{2}+\hat{p}_{i}^{2}\right)+\sinh \left(2 r_{\mathrm{b}}\right)\left(\hat{p}_{1} \hat{p}_{2}-\hat{q}_{1} \hat{q}_{2}\right),
$$

$$
\hat{H}_{\mathrm{f}}=\frac{\mathrm{i} \cos \left(2 r_{\mathrm{f}}\right)}{2} \sum_{i=1,2}\left(\hat{\gamma}_{i} \hat{\eta}_{i}-\hat{\eta}_{i} \hat{\gamma}_{i}\right)+\mathrm{i} \sin \left(2 r_{\mathrm{f}}\right)\left(\hat{\gamma}_{1} \eta_{2}-\gamma_{2} \eta_{1}\right)
$$

The ground states of these Hamiltonians are two-mode squeezed states. Accordingly, the identification maps $L_{1}$ and $L_{2}$ are represented by

$$
\left.\begin{array}{cc}
0 & 0 \\
0 & 0 \\
\cos \left(r_{\mathrm{f}}\right) \cosh \left(r_{\mathrm{b}}\right)-\sin \left(r_{\mathrm{f}}\right) \sinh \left(r_{\mathrm{b}}\right) & \cos \left(r_{\mathrm{f}}\right) \sinh \left(r_{\mathrm{b}}\right)-\sin \left(r_{\mathrm{f}}\right) \cosh \left(r_{\mathrm{b}}\right) \\
\cos \left(r_{\mathrm{f}}\right) \sinh \left(r_{\mathrm{b}}\right)+\sin \left(r_{\mathrm{f}}\right) \cosh \left(r_{\mathrm{b}}\right) & \sin \left(r_{\mathrm{f}}\right) \sinh \left(r_{\mathrm{b}}\right)+\cos \left(r_{\mathrm{f}}\right) \cosh \left(r_{\mathrm{b}}\right)
\end{array}\right),
$$

They lead to the complex structures

$$
\begin{array}{r}
J^{\mathrm{b}} \equiv\left(\begin{array}{cccc}
0 & 0 & \cosh \left(2 r_{\mathrm{b}}\right) & \sinh \left(2 r_{\mathrm{b}}\right) \\
0 & 0 & \sinh \left(2 r_{\mathrm{b}}\right) & \cosh \left(2 r_{\mathrm{b}}\right) \\
-\cosh \left(2 r_{\mathrm{b}}\right) & \sinh \left(2 r_{\mathrm{b}}\right) & 0 & 0 \\
\sinh \left(2 r_{\mathrm{b}}\right) & -\cosh \left(2 r_{\mathrm{b}}\right) & 0 & 0
\end{array}\right), \\
J^{\mathrm{f}} \equiv\left(\begin{array}{cccc}
0 & 0 & \cos \left(2 r_{\mathrm{f}}\right) & \sin \left(2 r_{\mathrm{f}}\right) \\
0 & 0 & -\sin \left(2 r_{\mathrm{f}}\right) & \cos \left(2 r_{\mathrm{f}}\right) \\
-\cos \left(2 r_{\mathrm{f}}\right) & \sin \left(2 r_{\mathrm{f}}\right) & 0 & 0 \\
-\sin \left(2 r_{\mathrm{f}}\right) & -\cos \left(2 r_{\mathrm{f}}\right) & 0 & 0
\end{array}\right),
\end{array}
$$

Let us now study how the identification maps act on the single site modes $\left(\hat{q}_{1}, \hat{p}_{1}\right)$ and $\left(\hat{\gamma}_{1}, \hat{\eta}_{1}\right)$, respectively. It is clear from Eq. (93) that, when both the bosonic and fermionic squeezing vanish, i.e., $r_{\mathrm{b}}=0=r_{\mathrm{f}}$, these are trivially identified with each other. However, when either squeezing parameter takes a nonzero value, the identification maps will mix the modes 1 and 2 .

Beginning with the bosonic mode $S=\left(\hat{q}_{1}, \hat{p}_{1}\right)$, we find it has the restricted complex linear structure

$$
J_{S}^{\mathrm{b}} \equiv\left(\begin{array}{cc}
0 & \cosh \left(2 r_{\mathrm{b}}\right) \\
-\cosh \left(2 r_{\mathrm{b}}\right) & 0
\end{array}\right)
$$

which define pure two-mode squeezed states. 
which has eigenvalues $\pm \mathrm{i} \cosh \left(2 r_{\mathrm{b}}\right)$, signaling that, for $r_{\mathrm{b}}>0$, the mode is in a mixed Gaussian state due to its entanglement with mode 2. Now we can use $L_{2}$ to associate this bosonic mode with a fermionic mode. Here, we need to consider that the fermionic observables $L_{2}\left(\hat{q}_{1}\right)$ and $L_{2}\left(\hat{p}_{1}\right)$ are not properly normalized Majorana operators. In fact, as a consequence of Eq. (71), we have

$$
\left\langle J_{\mathrm{f}}\left|\left\{L_{2}\left(\hat{q}_{1}\right), L_{2}\left(\hat{p}_{1}\right)\right\}\right| J_{\mathrm{f}}\right\rangle=\left\langle J_{\mathrm{b}}\left|\left\{\hat{q}_{1}, \hat{p}_{1}\right\}\right| J_{\mathrm{b}}\right\rangle=\cosh \left(2 r_{\mathrm{b}}\right) .
$$

Instead, the properly normalized Majorana operators, which correspond to an orthogonal basis in the fermionic phase space, are

$$
\tilde{L}_{2}\left(\hat{q}_{1}\right):=\frac{L_{2}\left(\hat{q}_{1}\right)}{\sqrt{\cosh \left(2 r_{\mathrm{b}}\right)}}, \quad \tilde{L}_{2}\left(\hat{p}_{1}\right):=\frac{L_{2}\left(\hat{p}_{1}\right)}{\sqrt{\cosh \left(2 r_{\mathrm{b}}\right)}},
$$

which we can use to calculate the restriction of $J_{\mathrm{f}}$ to this subsystem, e.g.,to calculate its entanglement with the rest of the fermionic system. When calculating their commutator, we can make use of Eq. (72) to find

$$
\begin{aligned}
& \left\langle J_{\mathrm{f}}\left|\tilde{L}_{2}\left(\hat{q}_{1}\right) \tilde{L}_{2}\left(\hat{p}_{1}\right)-\tilde{L}_{2}\left(\hat{p}_{1}\right) \tilde{L}_{2}\left(\hat{q}_{1}\right)\right| J_{\mathrm{f}}\right\rangle \\
& \quad=\frac{1}{\cosh \left(2 r_{\mathrm{b}}\right)}\left\langle J_{\mathrm{b}}\left|\hat{q}_{1} \hat{p}_{1}-\hat{p}_{1} \hat{q}_{1}\right| J_{\mathrm{b}}\right\rangle=\frac{\mathrm{i}}{\cosh \left(2 r_{\mathrm{b}}\right)} .
\end{aligned}
$$

Hence, the restriction of $J_{\mathrm{f}}$ has eigenvalues $\pm \mathrm{i}\left[\cosh \left(2 r_{\mathrm{b}}\right)\right]^{-1}$, as predicted by the entanglement duality.

The opposite identification of the fermionic mode $R=$ $\left(\hat{\gamma}_{1}, \hat{\eta}_{1}\right)$ with a bosonic mode is completely analogous, but it additionally highlights an important effect in the limit of $r_{\mathrm{f}} \rightarrow \pi / 4$. The restriction of $J_{\mathrm{f}}$ to $\left(\hat{\gamma}_{1}, \hat{\eta}_{1}\right)$ is represented by

$$
J_{R}^{\mathrm{f}} \equiv\left(\begin{array}{cc}
0 & \cos \left(2 r_{\mathrm{f}}\right) \\
-\cos \left(2 r_{\mathrm{f}}\right) & 0
\end{array}\right),
$$

which has eigenvalues $\pm \mathrm{i} \cos \left(2 r_{\mathrm{f}}\right)$. Mapping $\hat{\gamma}_{1}$ and $\hat{\eta}_{1}$ via the identification map $L_{1}$, we obtain two bosonic observables which obey

$$
\left\langle J_{\mathrm{b}}\left|\left[L_{1}\left(\hat{\gamma}_{1}\right), L_{1}\left(\hat{\eta}_{1}\right)\right]\right| J_{\mathrm{b}}\right\rangle=\cos \left(2 r_{\mathrm{f}}\right) .
$$

Hence, we need to rescale the operators

$$
\tilde{L}_{1}\left(\hat{\gamma}_{1}\right):=\frac{L_{1}\left(\hat{\gamma}_{1}\right)}{\sqrt{\cos \left(2 r_{\mathrm{f}}\right)}}, \quad \tilde{L}_{1}\left(\hat{\eta}_{1}\right):=\frac{L_{1}\left(\hat{\eta}_{1}\right)}{\sqrt{\cos \left(2 r_{\mathrm{f}}\right)}},
$$

to obtain properly anticommuting quadrature operators, defining a bosonic mode. For these, we find

$$
\left\langle J_{\mathrm{b}}\left|\left\{\tilde{L}_{1}\left(\hat{\gamma}_{1}\right), \tilde{L}_{1}\left(\hat{\eta}_{1}\right)\right\}\right| J_{\mathrm{b}}\right\rangle=\frac{1}{\cos \left(2 r_{\mathrm{f}}\right)},
$$

showing that the restriction of $J_{\mathrm{b}}$ has eigenvalues $\pm \mathrm{i}\left[\cos \left(2 r_{\mathrm{f}}\right)\right]^{-1}$.

In the limit of $r_{\mathrm{f}} \rightarrow \pi / 4$, the fermionic site mode $\left(\hat{\gamma}_{1}, \hat{\eta}_{1}\right)$ approaches maximal entanglement with the rest of the system, corresponding to an entanglement entropy of one bit, i.e., $\log 2$ in natural units. Consequently, also its bosonic dual system approaches maximal entanglement. However, for the bosonic mode, this means that its entanglement entropy grows without bound, as shown in Fig. 3. At the point of $r_{\mathrm{f}}=\pi / 4$, the fermionic mode 1 would represent a fermionic subsystem which is maximally entangled with mode 2 . However, such a fermionic mode is not mapped to a valid bosonic subsystem by the identification maps. In fact, for $r_{\mathrm{f}}=\pi / 4$, the identification map $L_{1}$ acts as

$$
\begin{aligned}
& L_{1}\left(\hat{\gamma}_{1}\right)=\frac{\cosh \left(r_{\mathrm{b}}\right)-\sinh \left(r_{\mathrm{b}}\right)}{\sqrt{2}}\left(\hat{q}_{1}+\hat{q}_{2}\right), \\
& L_{1}\left(\hat{\eta}_{1}\right)=\frac{\cosh \left(r_{\mathrm{b}}\right)-\sinh \left(r_{\mathrm{b}}\right)}{\sqrt{2}}\left(\hat{p}_{1}-\hat{p}_{2}\right),
\end{aligned}
$$

which are commuting observables, and thus do not define a proper bosonic mode [cf. Eq. (58)], as also seen by the fact that Eq. (101) vanishes.

This example highlights how, in general, fermionic Majorana operators that generate an almost maximally entangled mode are mapped to almost commuting bosonic operators by the identification maps, which in the limit of maximal fermionic entanglement, thus, fail to define a bosonic mode. The following Sec. IIIF showcases a peculiar consequence of this fundamental relationship between highly entangled fermionic modes and their bosonic counterparts in 2D.

\section{F. Application: supersymmetric Kitaev honeycomb model}

In this section, we demonstrate consequences of the derived entanglement duality in the example of the celebrated Kitaev honeycomb model [60], a spin model with characteristic bond-directional exchanges on the honeycomb lattice [Fig. 4(a)], and its supersymmetric extension [11]. In their gapped phases, both the fermionic and the bosonic lattice of this supersymmetric system exhibit the entanglement-area law in Eq. (2). Because the identification maps between the fermionic and the bosonic lattice behave local and preserve the shape of subregions of a lattice very well, one may expect also the entropy of these dual subsystems to follow an area law. However, we show that, in mapping from fermionic subregions to bosonic ones, a peculiar phenomenon can arise where the entanglement entropy of the dual bosonic subsystems scales much faster than its pre-image in the fermionic lattice which follows the area law. This is attributed to the presence of almost maximally entangled modes in the fermionic subsystem.

The analytical solution of the Kitaev honeycomb model is achieved by recasting it in terms of noninteracting Majorana fermions hopping on the same honeycomb lattice [in the background of a classical (static) $Z_{2}$ gauge field]. The resulting fermionic Hamiltonian reads

$$
\hat{H}_{\mathrm{f}}=-\frac{\mathrm{i}}{2} \sum_{i, j=1}^{N}\left(\hat{\eta}_{i} \mathcal{A}_{i j}^{\top} \hat{\gamma}_{j}-\hat{\gamma}_{i} \mathcal{A}_{i j} \hat{\eta}_{j}\right) .
$$

Expressed this way, $\hat{H}_{\mathrm{f}}$ describes the hopping of Majorana fermions between the two types of sites of the honeycomb lattice [Fig. 4(a)], where each of the Majorana operators $\hat{\gamma}_{i}$ and $\hat{\eta}_{i}$ resides on one type of the lattice sites. The $N \times N$-matrix $\mathcal{A}$ corresponds to the connectivity matrix of the lattice as depicted in Fig. 4(a), which we consider to be periodic. It involves the hopping strengths along the three bonds around each site of the honeycomb lattice, which we denote by $j_{x}, j_{y}, j_{z}$. The inequality $\left|j_{x}\right| \leqslant\left|j_{y}\right|+\left|j_{z}\right|$ and its cyclic permutations together imply a gapless spectrum of $\hat{H}_{\mathrm{f}}$; otherwise, $\hat{H}_{\mathrm{f}}$ has a gapped spectrum. While the phenomena discussed 


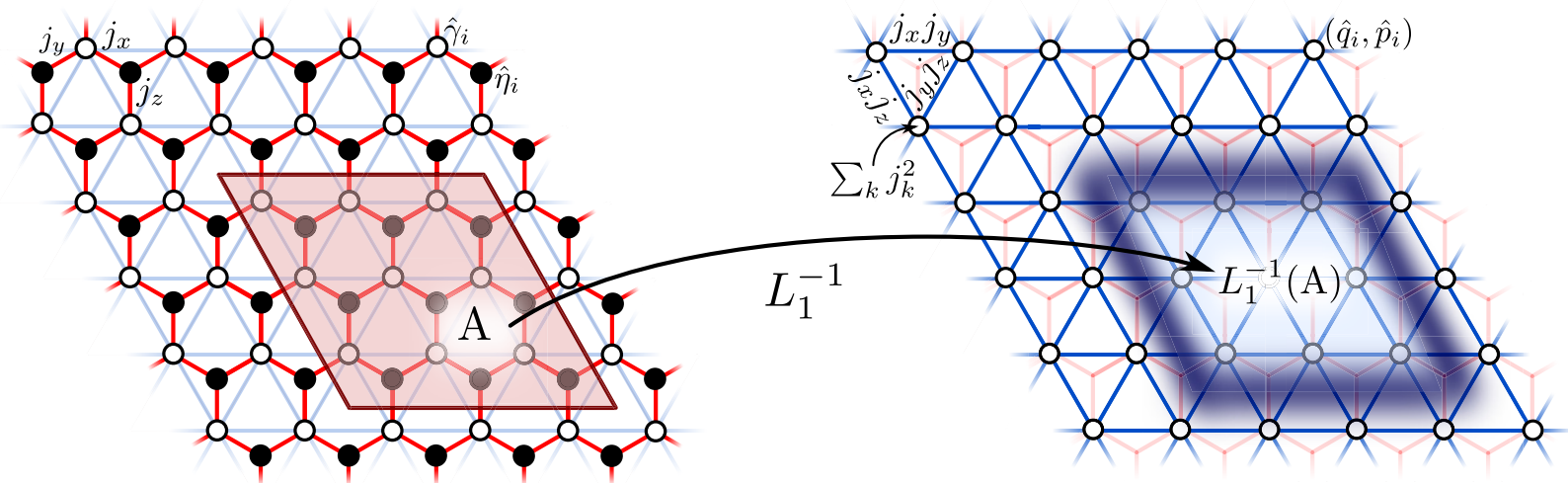

(a)

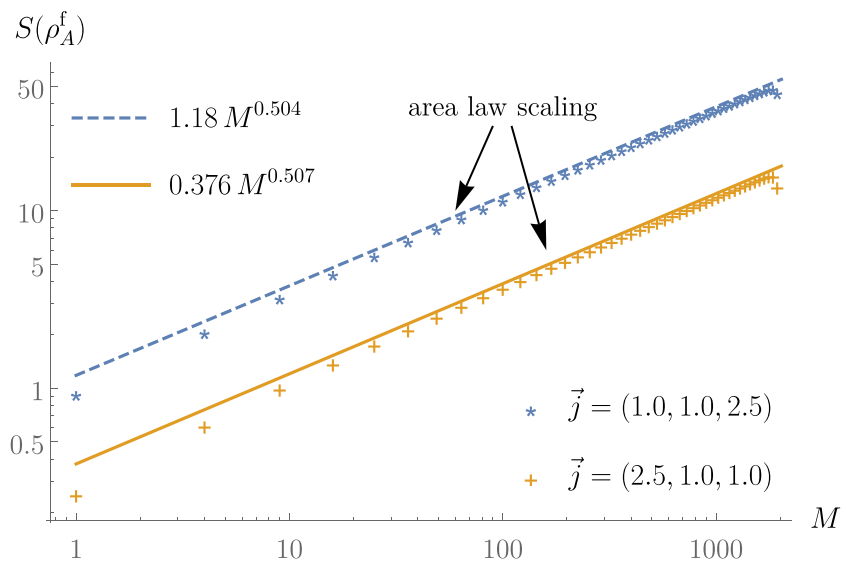

(b)

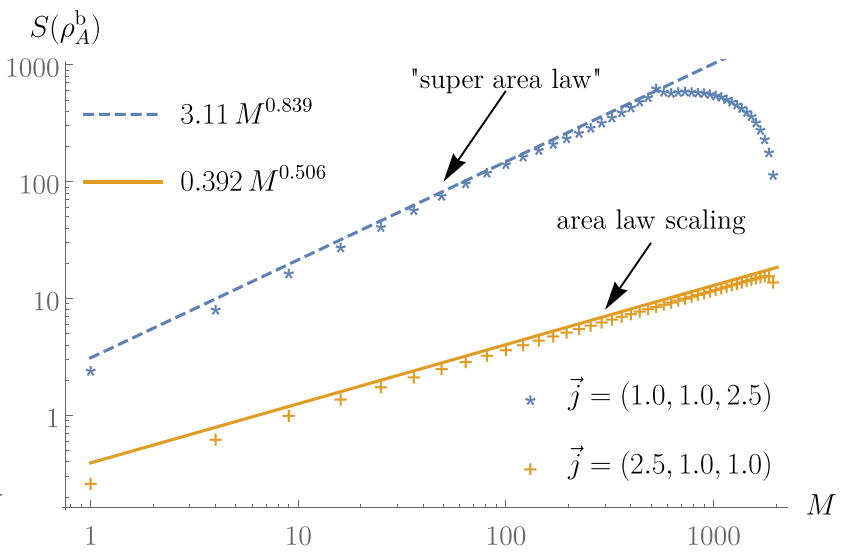

(c)

FIG. 4. Supersymmetric Kitaev honeycomb model: The schematic visualization in (a) represents, on the left, the fermionic honeycomb Kitaev model in Eq. (105) and, on the right, the bosonic triangular lattice in Eq. (107), which are generated by the supercharge in Eq. (106). The map $L_{1}^{-1}$ identifies fermionic subsystems with bosonic dual subsystems. The plots below, in (b) and (c), show numerical calculations of the entanglement entropy of parallelogram-shaped subsystems on the fermionic side and of their dual bosonic subsystems on the other side for two different orientations of the hopping parameters $\vec{j}=\left(j_{x}, j_{y}, j_{z}\right)$. For the numerical examples, periodic lattices with a total number of $N=45 \times 45=2025$ unit cells were considered. The parallelograms of the subsystems contain $M=m \times m$ modes, i.e., $M$ unit cells of the honeycomb lattice, and are oriented such that they do not cut through links with hopping parameter $j_{x}$. The fermionic entropies show good numerical agreement with the area law, which they are known to follow in the thermodynamic limit. Depending on the orientation of the couplings $\vec{j}$ relative to the parallelogram, the dual entropies can follow the area law or a scale much faster.

below can arise in both phases, for our numerical results, we will focus on the gapped phase below.

A supercharge operator $\hat{\mathcal{Q}}=R_{\alpha a} \hat{\xi}_{\mathrm{f}}^{\alpha} \hat{\xi}_{\mathrm{b}}^{a}$ that leads to $\hat{H}_{\mathrm{f}}$ being identified with the fermionic part of the supersymmetric Hamiltonian $\hat{H}=\hat{\mathcal{Q}}^{2}$ is [11]

$$
\hat{\mathcal{Q}}=\sum_{i, j=1}^{N}\left(\hat{\gamma}_{i} \mathcal{A}_{i j} \hat{q}_{j}+\hat{\eta}_{i} \delta_{i j} \hat{p}_{j}\right),
$$

which implies a block-diagonal matrix representation of $R_{\alpha a}$. The bosonic part of this Hamiltonian

$$
\hat{H}_{\mathrm{b}}=\frac{1}{2} \sum_{i, j=1}^{N} \hat{q}_{i}\left(\mathcal{A}^{\top} \mathcal{A}\right)_{i j} \hat{q}_{j}+\sum_{i=1}^{N} \hat{p}_{i}^{2},
$$

corresponds to a triangular lattice of harmonic oscillators, as depicted in Fig. 4(a), or in the appropriate classical limit, to a triangular network of balls and springs [11].

As previously mentioned, in the gapped phase, both the fermionic and the bosonic lattices of this SUSY Hamiltonian exhibit an area law scaling in Eq. (2) in the entanglement entropy of lattice subregions. Accordingly, Fig. 4(b) shows good agreement of our numerical example with an area law scaling of the entanglement entropy. There, we consider a honeycomb lattice which is periodic, with equal side lengths, comprising $N=45 \times 45=2025$ unit cells, of two sites each, in total. From this fermionic lattice, we cut out parallelogram-shaped subsystems of side length $m$, i.e.,containing $M=m \times m$ unit cells, as indicated in Fig. 4(a), and calculate their entanglement entropy $S\left(\rho_{A}^{f}\right)$ with the rest of the lattice. We compare two different combinations of the hoppings $\vec{j}=\left(j_{x}, j_{y}, j_{z}\right)=$ $(2.5,1,1)$ and $\vec{j}=(1,1,2.5)$ with respect to the orientation of the parallelograms. These two orientations differ in the type of neighboring sites which the boundary of the parallelogram separates.

We emphasize that the absolute shape of the boundary is not crucial here; instead, what types of links (strong or weak) are separated leaves remarkable effects on the scaling of the entanglement entropy in the dual bosonic subsystem, as will 
be demonstrated shortly. In detail, the boundary chosen here only cuts through links with hopping $j_{y}$ and $j_{z}$. Thus, in the first case, it only separates sites linked by the two weaker hoppings, whereas in the second case, it separates pairs of sites linked by the strong hopping. Since the entanglement area law holds independent of the orientation of the couplings, Fig. 4(b) shows that the fermionic entanglement does exhibit the scaling as predicted by the area law for both the orientations. The only visible difference is that, in the second case, due to the separation of strongly linked sites, the overall entanglement entropy is about three times larger.

However, one subtle difference between the two orientations which is not evident from the total fermionic entanglement entropy of the parallelogram subsystems is that, in the second case, when strongly linked sites are separated by the boundary of a subsystem, the subsystem develops a significant fraction of "almost maximally entangled" fermionic modes that scales as $\sqrt{M}$ for $M$ sites in the subsystem. However, as we shall see, these fermionic modes lead to a vastly different behavior of the entanglement entropy of the dual subsystems in the bosonic lattice associated with the original fermionic parallelograms by the identification maps.

In the periodic lattices considered here, the identification maps behave local in the sense that onsite operators in one lattice are mapped to exponentially localized operators on the supersymmetric partner lattice. Hence, the geometrical appearance of subsystems is well preserved when they are mapped to their dual subsystems in the supersymmetric partner lattice by the identification maps. At first sight, this may seem to suggest that the entanglement entropy of the dual systems also should exhibit an area law scaling since the entanglement area relation of Eq. (2) holds in both the fermionic and the bosonic lattice we consider. However, Fig. 4(c) demonstrates, for the numerical example introduced before, that the entanglement entropy of fermionic subsystems and their dual bosonic subsystems can scale very differently: depending on the hoppings $\vec{j}$ with respect to the parallelogram subsystems, the dual entropy may scale in agreement with an area law scaling, or they can scale much faster according to a "super area law."

How does this phenomenon arise? First, let us note that, when the parallelogram in the original fermionic lattice only cuts links with weaker hoppings, the dual entropy follows an area law scaling. The higher scaling of the dual entropy appears when the parallelogram cuts through links with the strongest hopping. This separation of strongly linked Majorona sites, however, heralds the presence of normal modes in the fermionic parallelogram which are (almost) maximally entangled with the rest of the lattice. The presence of such modes is the reason for the observed peculiar scaling of the dual entropies.

In fact, the mathematical explanation for the observed amplified scaling of the dual entropies is rooted in the spectrum of the restricted fermionic linear complex structure $J_{A}^{\mathrm{f}}$. Figure 5 plots the absolute values of the eigenvalues for the subsystems considered in Fig. 4(a) for the two distinct orientations of the hopping mentioned before. In the first case, where the parallelograms do not cut through any strong links, the eigenvalues roughly lie in the interval $0.9<\left|\lambda_{i}\right| \leqslant 1$, as seen in the inset of Fig. 5. As is evident from Fig. 3, in this regime,

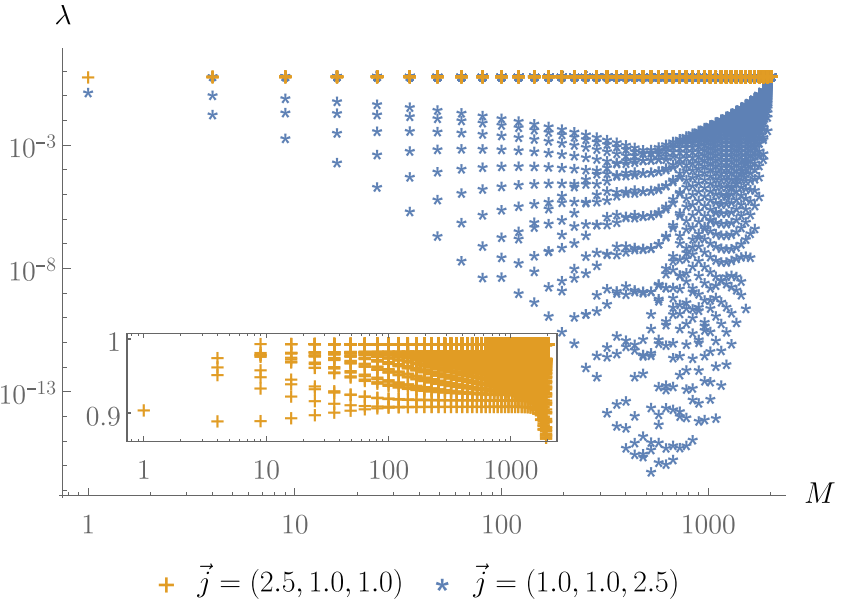

FIG. 5. Absolute values of the eigenvalues of the fermionic linear complex structure $J_{A}^{\mathrm{f}}$ on the Kitaev honeycomb lattice restricted to the subsystems of Fig. 4. The inset zooms in on the eigenvalues of the first case $\vec{j}=(2.5,1,1)$, which hardly fall below $\left|\lambda_{i}\right| \approx 0.9$. In the second case $\vec{j}=(1,1,2.5)$, the absolute values decay exponentially with the subsystem size $M$, thus triggering the amplified scaling of the dual entropies in Fig. 4(c) up until the subsystem exhausts the full lattice of $N=2025$ modes.

the entanglement entropy of each of the eigenmodes of $J_{A}^{\mathrm{f}}$, i.e.,the normal modes of the subsystem, is almost the same as the entanglement entropy of their dual bosonic modes. Thus, in the first case, the entanglement entropies for the fermionic subsystems and their bosonic duals are almost the same and follow the same scaling.

In contrast, in the second case, the spectrum of $J_{A}^{\mathrm{f}}$ exhibits a certain number of eigenvalues which are very small or almost zero. Note that the number of these pairs of eigenvalues $\pm \mathrm{i} \lambda_{i}$ that fall below $\lambda_{i} \lesssim 0.1$ corresponds exactly to the side length of the parallelograms, i.e.,is half of the number of strong links which the parallelogram cut through. The normal modes corresponding to these eigenvalues thus share almost maximal entanglement with the rest of the system, i.e.,the complement of the region surrounded by the parallelogram. In terms of their entanglement entropy, as discussed before and also evident from Fig. 3, the fermionic normal modes approach the maximum value of one bit entanglement entropy as $\lambda_{i} \rightarrow 0$ following Eq. (65), whereas the entanglement entropy of their dual bosonic modes diverges as $\lambda_{i}^{-1} \rightarrow \infty$.

As a result, the total entanglement entropy of the dual bosonic subsystem scales much faster with its subsystem size than the original fermionic system does. This effect is visualized in Fig. 6, whose stacked plots show the mode-wise contribution of the normal modes to the total entropy of the fermionic subregions and their duals in the bosonic lattice. On the fermionic side, the individual contributions are bounded by one bit per mode; thus, their summed contribution still results in a growth linear in the perimeter of the parallelogram. However, on the bosonic side, the individual contribution from each normal mode continues to grow as the system size increases, resulting in a higher scaling of the entanglement entropy than that predicted by the area law. Let us emphasize that the total number of low-lying fermionic eigenvalues scaling as 

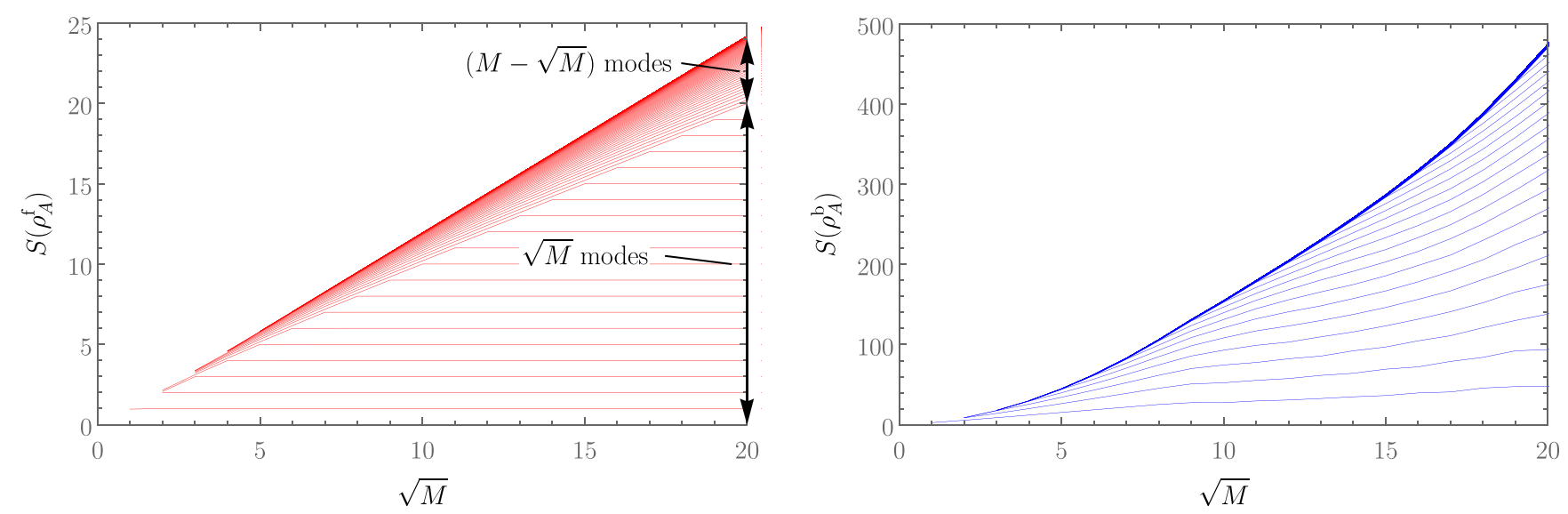

FIG. 6. Stacked plots of the mode-wise contribution to the total entanglement entropy, for the setup of Figs. 4(b) and 4(c), for the case $\vec{j}=(1,1,2.5)$. The left plot refers to the fermionic hexagonal subsystems $A_{\mathrm{f}}$ and the right plot to the dual bosonic subsystem $L_{1}^{-1}\left(A_{\mathrm{f}}\right)$. The difference between the $(i-1)$ th and $i$ th line gives the contribution of the $i$ th normal mode of the subsystem to the total entanglement entropy. Thus, the upper-most line coincides with the respective plot in Figs. 4(b) and 4(c). Note that, here, we have changed the horizontal axis to $\sqrt{M}$, i.e.,the side length of the parallelogram-shaped fermionic subsystems. The fermionic entropy is dominated by the $\sqrt{M}$ normal modes that are almost maximally entangled and the bosonic entropy by their dual modes.

$\sqrt{M}$ (with $M$ being the subsystem size) alone is not sufficient to give rise to a "super area law" on the bosonic side but also that these low-lying values actually decay towards zero. If they were bounded by some $\lambda_{\min }$, such that $\lambda_{i} \geqslant \lambda_{\min }>0$, the entropy of each dual bosonic mode would be upper bounded by $s_{\mathrm{b}}\left(1 / \lambda_{\min }\right)$, resulting again in the conventional $\sqrt{M}$ scaling of the area law.

The peculiar phenomenon observed above can be viewed as a direct physical instance of the minimal two-mode example in Sec. IIIE taking place at the edge of the subsystem: Every time its boundary cuts through a pair of strong links (on opposite sites of the parallelogram cutout), the subsystem exhibits a strongly entangled normal mode (corresponding to an almost vanishing eigenvalue of the restricted complex structure). These normal modes are highly localized at the edge of the subsystem and share no entanglement with any mode inside the subsystem but with those lying on the complement of the subsystem. In fact, the normal modes of the subsystem, which carry entanglement, form pairs with those from the complement such that each normal mode is entangled with exactly one partner (normal) mode of the complement. ${ }^{10}$ The partner normal modes of the highly entangled subsystem normal modes are localized right outside the subsystem. Thus, a pair of partner modes (one inside the subsystem and one outside) forms a two-mode subsystem, localized in the immediate neighborhood of the boundary of the subsystem, which is not entangled with the rest of the system but in a pure two-mode squeezed state on its own. The identification maps now map each pair of such fermionic normal mode partners to a pair of bosonic normal mode partners, one inside the dual subsystem and one outside. Due to the locality properties of the identification maps, their joint support on the bosonic lattice sites is closely related to the shape of the fermionic pair.

\footnotetext{
${ }^{10}$ These pairs are connected by the complex structure $J_{\mathrm{f}}$ of the ground state.
}

In this mapping, pair by pair, the same mechanism as discussed in Eq. (104) takes place. The Majorana operators of the fermionic subsystem normal mode are mapped to a pair of bosonic observables which are almost commuting, thus defining a highly entangled bosonic mode. Such almost commuting bosonic observables need not be spatially separated on the lattice, but they can have equal support on the same lattice sites, as Eq. (104) demonstrates: There, both bosonic observables have equal support on both of the two modes; however, one quadrature is proportional to $\hat{q}_{1}+\hat{q}_{2}$ but the other to $\hat{p}_{1}-\hat{p}_{2}$; thus, they commute.

Because of such localized and highly entangled bosonic modes, it is possible for the dual bosonic subsystems, despite being well localized, to exhibit a scaling of entanglement entropy that exceeds the area law of the original fermionic lattice. The entanglement area law assumes the subsystem division being a direct sum of individual lattice sites, i.e.,in a bosonic system, the quadarature operators $\hat{q}_{i}$ and $\hat{p}_{i}$ either both belong to the subsystem, or they both do not. In contrast, the boundary between the dual bosonic subsystems and the rest of the (bosonic) lattice considered in this example may well separate different linear combinations of the onsite bosonic operators.

\section{DISCUSSION}

In this paper, we study the entanglement properties of bosonic and fermionic Gaussian states that are related via SUSY, in other words, belong to Hamiltonians which are supersymmetric partners of each other. After reviewing a unified framework to describe these states in terms of Kähler structures, we prove the main result of this paper in Proposition 1, which relates the bosonic and the fermionic entanglement spectrum of a chosen subsystem in a supersymmetric Gaussian state. The result is based on supersymmetric identification maps that are constructed from the supercharge operator $\hat{\mathcal{Q}}$. They enable us to uniquely identify subsystems 
both bosonic and fermionic, which we refer to as dual to each other. In Proposition 2, we extend the said duality to include thermal states associated with supersymmetric Hamiltonians, for which we find the same relationship between the bosonic and the fermionic thermal states as for the reduced states in the subsystems.

The rest of this paper illustrates this result and its implications in supersymmetric lattice models. We investigate to what extent identification maps constructed from a local supercharge operator preserve this locality, i.e.,to what extent a local subsystem on the bosonic side is identified with a local subsystem on the fermionic side and vice versa. This is important to explain why our abstract duality is of relevance when studying physical systems: It shows a simple relation between the entanglement of bosonic and fermionic subsystems that can both be thought local in a precise way.

The examples in this paper suggest that, for SUSY lattice Hamiltonians, the locality properties of the identification maps are related to the boundary conditions of the lattice and the presence of edge modes. For example, in the 1D open chain of Sec. IID, the identification maps featured highly nonlocal behavior in the topological phase. However, in the same system with periodic boundary conditions [obtained by extending the supercharge in Eq. (54) to be translation invariant], the identification maps behave rather local, even deep in the topological phase.

In the context of localized subsystems, a peculiar consequence of the entanglement duality is the appearance of "super area law" behavior in the entanglement entropy of bosonic subsystems dual to subsystems with certain shapes on the fermionic side, seen in Sec. IIIF. This phenomenon is related to the appearance of almost maximally entangled modes in the fermionic subsystem, for which the spectrum of the fermionic linear complex structure nearly vanishes. The entanglement duality then implies an unbounded growth of entanglement for the dual bosonic system. Since it is well known [61] that the entanglement entropy associated with a ground state of a gapped and local Hamiltonian (bosonic or fermionic) satisfies an area law, this raises the question of how occasions where our entanglement duality relates an area law on the fermionic side with a "super area law" on the bosonic side for the respective ground states of a local supersymmetric Hamiltonian (such as the honeycomb model considered in III F) can appear. The answer to this question lies in the type of bosonic subsystem that arises under the duality for a fermionic subsystem with large entanglement. We saw in Sec. III E how there can be an arbitrary amount of entanglement associated with a single bosonic mode due to choosing a subsystem that effectively partially separates a quadrature operator $\hat{q}_{i}$ from its canonically conjugate operator $\hat{p}_{i}$. Such types of subsystems are typically not considered in the context of studying area laws, and it is not surprising that the standard results on area laws for the ground states of gapped local Hamiltonians do not apply to them.

At this stage, it is a natural question to what extent such dual subsystems, identified by the supersymmetric duality as in Sec. IIIF, should be considered a physical reality or a mathematical concept. The answer will highly depend on the concrete physical realization of the model. For example, if the bosonic degrees of freedom were represented by photons, such a subsystem could at least in principle be probed by applying an appropriate sequence of Gaussian transformations (implemented by standard linear optics devices) as long as $\hat{q}_{i}$ and $\hat{p}_{i}$ are not fully separated, i.e., $\left[\hat{q}_{i}, \hat{p}_{i}\right] \neq 0$. On the other hand, since the implementation of large squeezing, as discussed in Sec. IIIE, is known to be very challenging, accessing the subsystems may well be physically infeasible.

In keeping with the study of topological properties of translation-invariant SUSY lattice Hamiltonians in arbitrary dimensions, a generalization of the identification maps to higher dimensional lattices certainly constitutes a promising avenue to explore. Here, we would like to highlight Ref. [71], where a spin-fermion correspondence, very much in the same spirit of our SUSY map, has been worked out engaging three-dimensional (3D) lattice models as well. Entanglement properties of a 3D generalization of the Kitaev honeycomb model have also been studied [72]. Other variants of 3D Kitaev spin liquids exist [73,74] with entanglement properties hitherto unexplored; our dualities find a straightforward application therein.

\section{ACKNOWLEDGMENTS}

R.H.J. gratefully acknowledges support by the WennerGren Foundations. L.H. gratefully acknowledges financial support by the Alexander von Humboldt Foundation. K.R. thanks the sponsorship, in part, by the Swedish Research Council.
[1] Y. A. Gol'fand and E. P. Likhtman, Extension of the algebra of Poincaré group generators and violation of $\mathrm{P}$ invariance, in $\mathrm{Su}$ pergravities in Diverse Dimensions (World Scientific Publishing Company, Teaneck, 1989), Vol. 1, pp. 20-23.

[2] P. Ramond, Dual theory for free fermions, Phys. Rev. D 3, 2415 (1971).

[3] A. Neveu and J. H. Schwarz, Factorizable dual model of pions, Nucl. Phys. B 31, 86 (1971).

[4] N. Sourlas, Introduction to supersymmetry in condensed matter physics, Physica D 15, 115 (1985).

[5] H. Baer and X. Tata, Weak Scale Supersymmetry: From Superfields to Scattering Events (Cambridge University Press, Cambridge, 2006).
[6] F. Cooper and B. Freedman, Aspects of supersymmetric quantum mechanics, Ann. Phys. 146, 262 (1983).

[7] F. Cooper, A. Khare, and U. Sukhatme, Supersymmetry and quantum mechanics, Phys. Rep. 251, 267 (1995).

[8] A. Kirchberg, J. D. Länge, P. A. G. Pisani, and A. Wipf, Algebraic solution of the supersymmetric hydrogen atom in $d$ dimensions, Ann. Phys. 303, 359 (2003).

[9] A. Gangopadhyaya, J. V. Mallow, and C. Rasinariu, Supersymmetric Quantum Mechanics: An Introduction (World Scientific Publishing Company, Singapore, 2017).

[10] M. J. Lawler, Supersymmetry protected topological phases of isostatic lattices and kagome antiferromagnets, Phys. Rev. B 94, 165101 (2016). 
[11] J. Attig, K. Roychowdhury, M. J. Lawler, and S. Trebst, Topological mechanics from supersymmetry, Phys. Rev. Res. 1, 032047(R) (2019).

[12] E. Witten, Constraints on supersymmetry breaking, Nucl. Phys. B 202, 253 (1982).

[13] C. L. Kane and T. C. Lubensky, Topological boundary modes in isostatic lattices, Nat. Phys. 10, 39 (2014).

[14] X.-B. Wang, T. Hiroshima, A. Tomita, and M. Hayashi, Quantum information with Gaussian states, Phys. Rep. 448, 1 (2007).

[15] C. Weedbrook, S. Pirandola, R. García-Patrón, N. J. Cerf, T. C. Ralph, J. H. Shapiro, and S. Lloyd, Gaussian quantum information, Rev. Mod. Phys. 84, 621 (2012).

[16] G. Adesso, S. Ragy, and A. R. Lee, Continuous variable quantum information: Gaussian states and beyond, Open Syst. Inf. Dyn. 21, 1440001 (2014).

[17] T. Shi, E. Demler, and J. I. Cirac, Variational study of fermionic and bosonic systems with non-Gaussian states: theory and applications, Ann. Phys. 390, 245 (2018).

[18] J. Dereziński and C. Gérard, Mathematics of Quantization and Quantum Fields, Cambridge Monographs on Mathematical Physics (Cambridge University Press, Cambridge, 2013).

[19] J. M. Deutsch, Quantum statistical mechanics in a closed system, Phys. Rev. A 43, 2046 (1991).

[20] M. Srednicki, Chaos and quantum thermalization, Phys. Rev. E 50, 888 (1994).

[21] M. Rigol, V. Dunjko, and M. Olshanii, Thermalization and its mechanism for generic isolated quantum systems, Nature (London) 452, 854 (2008).

[22] L. D'Alessio, Y. Kafri, A. Polkovnikov, and M. Rigol, From quantum chaos and eigenstate thermalization to statistical mechanics and thermodynamics, Adv. Phys. 65, 239 (2016).

[23] C. Gogolin and J. Eisert, Equilibration, thermalisation, and the emergence of statistical mechanics in closed quantum systems, Rep. Prog. Phys. 79, 056001 (2016).

[24] J. M. Deutsch, Eigenstate thermalization hypothesis, Rep. Prog. Phys. 81, 082001 (2018).

[25] S. Goldstein, J. L. Lebowitz, R. Tumulka, and N. Zanghì, Canonical Typicality, Phys. Rev. Lett. 96, 050403 (2006).

[26] S. Popescu, A. J. Short, and A. Winter, Entanglement and the foundations of statistical mechanics, Nat. Phys. 2, 754 (2006).

[27] H. Tasaki, From Quantum Dynamics to the Canonical Distribution: General Picture and a Rigorous Example, Phys. Rev. Lett. 80, 1373 (1998).

[28] A. Polkovnikov, K. Sengupta, A. Silva, and M. Vengalattore, Colloquium: Nonequilibrium dynamics of closed interacting quantum systems, Rev. Mod. Phys. 83, 863 (2011).

[29] L. Vidmar, L. Hackl, E. Bianchi, and M. Rigol, Entanglement Entropy of Eigenstates of Quadratic Fermionic Hamiltonians, Phys. Rev. Lett. 119, 020601 (2017).

[30] C. Liu, X. Chen, and L. Balents, Quantum entanglement of the Sachdev-Ye-Kitaev models, Phys. Rev. B 97, 245126 (2018).

[31] L. Vidmar, L. Hackl, E. Bianchi, and M. Rigol, Volume Law and Quantum Criticality in the Entanglement Entropy of Excited Eigenstates of the Quantum Ising Model, Phys. Rev. Lett. 121, 220602 (2018).

[32] L. Hackl, L. Vidmar, M. Rigol, and E. Bianchi, Average eigenstate entanglement entropy of the XY chain in a transverse field and its universality for translationally invariant quadratic fermionic models, Phys. Rev. B 99, 075123 (2019).
[33] L. Vidmar and M. Rigol, Entanglement Entropy of Eigenstates of Quantum Chaotic Hamiltonians, Phys. Rev. Lett. 119, 220603 (2017).

[34] E. Bianchi and P. Donà, Typical entanglement entropy in the presence of a center: Page curve and its variance, Phys. Rev. D 100, 105010 (2019).

[35] P. Łydżba, M. Rigol, and L. Vidmar, Eigenstate Entanglement Entropy in Random Quadratic Hamiltonians, Phys. Rev. Lett. 125, 180604 (2020).

[36] C. H. Bennett and P. W. Shor, Quantum information theory, IEEE Trans. Inf. Theory 44, 2724 (1998).

[37] J. Eisert, Entanglement in quantum information theory, Ph.D. thesis, University of Potsdam, 2001.

[38] P. Hayden, D. W. Leung, and A. Winter, Aspects of generic entanglement, Commun. Math. Phys. 265, 95 (2006).

[39] P. Hayden and J. Preskill, Black holes as mirrors: quantum information in random subsystems, J. High Energy Phys. 09 (2007) 120

[40] Y. Sekino and L. Susskind, Fast scramblers, J. High Energy Phys. 10 (2008) 065.

[41] P. Hosur, X.-L. Qi, D. A. Roberts, and B. Yoshida, Chaos in quantum channels, J. High Energy Phys. 02 (2016) 004.

[42] D. A. Roberts and B. Yoshida, Chaos and complexity by design, J. High Energy Phys. 04 (2017) 121.

[43] Y. O. Nakagawa, M. Watanabe, S. Sugiura, and H. Fujita, Universality in volume-law entanglement of scrambled pure quantum states, Nat. Commun. 9, 1635 (2018).

[44] T.-C. Lu and T. Grover, Renyi entropy of chaotic eigenstates, Phys. Rev. E 99, 032111 (2019).

[45] H. Fujita, Y. O. Nakagawa, S. Sugiura, and M. Watanabe, Page curves for general interacting systems, J. High Energy Phys. 12 (2018) 112.

[46] A. Kitaev and J. Preskill, Topological Entanglement Entropy, Phys. Rev. Lett. 96, 110404 (2006).

[47] M. Levin and X.-G. Wen, Detecting Topological Order in a Ground State Wave Function, Phys. Rev. Lett. 96, 110405 (2006).

[48] S. Furukawa and G. Misguich, Topological entanglement entropy in the quantum dimer model on the triangular lattice, Phys. Rev. B 75, 214407 (2007).

[49] H. Yao and X.-L. Qi, Entanglement Entropy and Entanglement Spectrum of the Kitaev Model, Phys. Rev. Lett. 105, 080501 (2010).

[50] S. V. Isakov, M. B. Hastings, and R. G. Melko, Topological entanglement entropy of a Bose-Hubbard spin liquid, Nat. Phys. 7, 772 (2011).

[51] S. Depenbrock, I. P. McCulloch, and Ulrich Schollwöck, Nature of the Spin-Liquid Qround State of the $S=1 / 2$ Heisenberg Model on the Kagome Lattice, Phys. Rev. Lett. 109, 067201 (2012).

[52] H.-C. Jiang, Z. Wang, and L. Balents, Identifying topological order by entanglement entropy, Nat. Phys. 8, 902 (2012).

[53] S.-S. Gong, W. Zhu, and D. N. Sheng, Emergent chiral spin liquid: fractional quantum Hall effect in a kagome Heisenberg model, Sci. Rep. 4, 6317 (2014).

[54] K. Roychowdhury, S. Bhattacharjee, and F. Pollmann, $\mathbb{Z}_{2}$ topological liquid of hard-core bosons on a kagome lattice at $1 / 3$ filling, Phys. Rev. B 92, 075141 (2015). 
[55] R. D. Sorkin, in General Relativity and Gravitation, proceedings of the GR10 Conference, Padova, 1983, edited by B. Bertotti, F. de Felice, and A. Pascolini (Roma, Consiglio Nazionale Delle Ricerche, 1983), Vol. II, pp. 734-736.

[56] I. Peschel, Calculation of reduced density matrices from correlation functions, J. Phys. A 36, L205 (2003).

[57] R. Horodecki, P. Horodecki, M. Horodecki, and K. Horodecki, Quantum entanglement, Rev. Mod. Phys. 81, 865 (2009).

[58] N. Laflorencie, Quantum entanglement in condensed matter systems, Phys. Rep. 646, 1 (2016).

[59] I. Bengtsson and K. Życzkowski, Geometry of Quantum States: An Introduction to Quantum Entanglement (Cambridge University Press, Cambridge, 2017).

[60] A. Kitaev, Anyons in an exactly solved model and beyond, Ann. Phys. 321, 2 (2006).

[61] J. Eisert, M. Cramer, and M. B. Plenio, Colloquium: Area laws for the entanglement entropy, Rev. Mod. Phys. 82, 277 (2010).

[62] L. Hackl and R. H. Jonsson, Minimal energy cost of entanglement extraction, Quantum 3, 165 (2019).

[63] L. Hackl and E. Bianchi, Bosonic and fermionic Gaussian states from Kähler structures, arXiv:2010.15518.

[64] B. Windt, A. Jahn, J. Eisert, and L. Hackl, Local optimization on pure Gaussian state manifolds, SciPost Phys. 10, 066 (2021).

[65] R. Penrose and W. Rindler, Spinors and Space-Time: Volume 1, Two-Spinor Calculus and Relativistic Fields (Cambridge University Press, Cambridge, 1984).
[66] E. Bianchi, L. Hackl, and N. Yokomizo, Linear growth of the entanglement entropy and the Kolmogorov-Sinai rate, J. High Energy Phys. 03 (2018) 025.

[67] A. Yu. Kitaev, Unpaired Majorana fermions in quantum wires, Phys. Usp. 44, 131 (2001).

[68] B. Gin-ge Chen, N. Upadhyaya, and V. Vitelli, Nonlinear conduction via solitons in a topological mechanical insulator, Proc. Natl. Acad. Sci. USA 111, 13004 (2014).

[69] L. Hackl, T. Guaita, T. Shi, J. Haegeman, E. A. Demler, and J. I. Cirac, Geometry of variational methods: dynamics of closed quantum systems, SciPost Phys. 9, 048 (2020).

[70] E. Bianchi, L. Hackl, and N. Yokomizo, Entanglement entropy of squeezed vacua on a lattice, Phys. Rev. D 92, 085045 (2015).

[71] J. Attig and S. Trebst, Classical spin spirals in frustrated magnets from free-fermion band topology, Phys. Rev. B 96, 085145 (2017).

[72] I. Mondragon-Shem and T. L. Hughes, Entanglement of a 3D generalization of the Kitaev model on the diamond lattice, J. Stat. Mech. (2014) P10022.

[73] K. O'Brien, M. Hermanns, and S. Trebst, Classification of gapless $\mathbb{Z}_{2}$ spin liquids in three-dimensional Kitaev models, Phys. Rev. B 93, 085101 (2016).

[74] T. Eschmann, P. A. Mishchenko, Kevin O’Brien, T. A. Bojesen, Y. Kato, M. Hermanns, Y. Motome, and S. Trebst, Thermodynamic classification of three-dimensional kitaev spin liquids, Phys. Rev. B 102, 075125 (2020). 